Research Article

\title{
Study on Optimal Dispatching Strategy of Regional Energy Microgrid
}

\author{
K. H. Wu $\mathbb{D}^{\text {, J. Wu }} \mathbb{D}^{\circ}$, L. Feng, B. Yang, R. Liang $\mathbb{D}^{\circ}$, S. Q. Yang $\mathbb{D}^{\circ}$, and R. Zhao \\ Economic \& Technology Research Institute State Grid Shandong Electric Power Company, Jinan 250021, China \\ Correspondence should be addressed to K. H. Wu; wukuihua123@outlook.com
}

Received 21 June 2020; Revised 14 August 2020; Accepted 24 September 2020; Published 27 October 2020

Academic Editor: Ying-Yi Hong

Copyright $\odot 2020 \mathrm{~K}$. H. Wu et al. This is an open access article distributed under the Creative Commons Attribution License, which permits unrestricted use, distribution, and reproduction in any medium, provided the original work is properly cited.

Considering the fluctuation of microgrid output and customer's demand, an optimal dispatching strategy for the combined cooling, heating, and power supply microgrid is proposed. The fluctuation of energy sources, such as a photovoltaic system and multiple loads, may affect the safety, economics, and stability in combined cooling, heating, and power microgrid operation. Therefore, the extreme learning machine optimized by particle swarm algorithm is used to improve the prediction accuracy of photovoltaic power generation, wind power generation, and load power. The regularization coefficient $C$ and the kernel parameter $\lambda$ of kernel extreme learning machine are regarded as the optimization targets of the particle swarm algorithm so that the prediction accuracy can be improved. Forecasted value of cooling, heating, and electricity microgrid system and new energy power generation as well as real-time electricity price, fuel unit price, etc. are considered in the operating cost. In order to minimize the operating cost and improve the energy utilization, an improved shuffled frog leaping algorithm is used to solve the cost minimization problem to give the equipment output dispatch strategy. Comparative simulation results can be found that under the same conditions, compared to the kernel extreme learning machine and the kernel extreme learning machine optimized by the genetic algorithm, the kernel extreme learning machine optimized by the particle swarm has faster convergence speed and higher prediction accuracy. Comparative simulations of microgrid dispatching on typical days in summer and winter are carried out. Compared with the cost of distribution, the cooling, heating, and power microgrid based on the improved shuffled frog leaping algorithm has obvious economic benefits and higher energy utilization property.

\section{Introduction}

In recent years, distributed energy systems have developed rapidly, and combined cooling, heating, and power (CCHP) systems have become a key solution to improve energy efficiency and reduce greenhouse gas emissions. Power generation, cooling, and heating capabilities are included in the CCHP microgrid at the same time. In the CCHP system, the energy of the micro power supply can be used in multiple levels, and the energy utilization efficiency can be improved $[1,2]$. Research on the CCHP microgrid is of great significance for the coordinated operation of multiple energy systems and improving the economic and environmental protection of the microgrid.

Economic performance is an important technical indicator of CCHP microgrid, so the optimal dispatch of CCHP has been paid more attention $[3,4]$. In [5], genetic algorithm is used to optimize the device output of the combined cooling, heating, and power microgrid; unfortunately, the uncertainty of microgrid output and load has not been considered when the CCHP optimization model is established. In [6], an optimal operation method of CCHP microgrid with the consideration of wind power uncertainties is designed; however, if the uncertainty of solar power generation and load in microgrids can be taken into account, energy efficiency will be further improved. The evaluation method of new energy optimal scheduling strategy was proposed in $[7,8]$, and the Monte Carlo method and analytic network process are used to evaluate the scheme to obtain an ideal scheduling scheme. Unfortunately, microgrid output and consumer demand are not taken into account when scheduling schemes are set up. In [9], the microgrid uses a scheme based on solar PV in addition to 
diesel generators and an energy storage system based on electrochemical batteries to increase the resilience of a microgrid feeding critical facility; however, the uncertainty of PV generation and consumer load are not considered in the dispatching of the microgrid. In [10], the scheduling problem of distributed energy resources is studied from various aspects such as modelling techniques, solving methods, reliability, emission, uncertainty, stability, demand response, and multiobjective standpoint in the microgrid and virtual power plant frameworks. Unfortunately, the uncertainty about consumer demand and microgrid output is not discussed. It is well known that energy management efficiency can be improved if the output of microgrid side and the demand of consumer side can be determined.

It is difficult to forecast wind, solar power generation, and load power in CCHP microgrid, which has attracted more and more attention. In [11], a load forecasting method based on an improved deep recurrent neural network is proposed, and a microgrid optimal scheduling model is established to reduce costs. However, the RMSE of this method is 7.58\%, which is still not ideal. In [12], a PV and wind output power generation forecasting method in smart microgrid is proposed. Based on an adaptive neurofuzzy inference system-based forecasting model, the forecast accuracy of photovoltaic and wind power has been improved. But the RMSE of the proposed method is higher than $2.8 \%$. In [13], load signal is decomposed by empirical mode decomposition, and adaptive network-based fuzzy inference systems optimized by particle swarm optimization is developed to forecast the short-term load. In [14], a new bilevel prediction strategy is proposed for short-term load forecast of microgrids; however, excessive amounts of computation time are a disadvantage. In [15], fuzzy prediction interval models are proposed for the microgrid energy management system; as we all know, complex computation is an inevitable problem of model predictive control.

On the one hand, accurate microgrid output and consumer load are important guarantees for the CCHP system. Therefore, kernel extreme learning machine-optimized particle swarm optimization is designed to improve the forecasting accuracy of the microgrid output and the customer load. On the other hand, in order to minimize the operating cost and improve the energy utilization, an improved shuffled frog leaping algorithm is used to solve the cost minimization problem to give the equipment output dispatch strategy.

This paper is organized as follows. The research status of the cooling, heating, and power system is studied in Section 1. In Section 2, the mathematical model of CCHP microgrids is established. In Section 3, kernel extreme learning machine-based particle swarm optimization is proposed, and the proposed algorithm is applied to forecast the photovoltaic generation and load power. In Section 4, the dispatch model with the minimum operating cost of the CCHP system is established, and the improved shuffled frog hop algorithm is used to optimize the dispatch. In Section 5, comparative simulation is established to verify the effectiveness of the proposed method. Section 6 is a summary.

\section{Mathematical Model of CCHP}

2.1. CCHP Microgrid Power Supply Structure. The energy supply structure of CCHP microgrid is shown in Figure 1.

As can be seen from Figure 1, electric load is mainly supplied by the fan, pumped storage, photovoltaic power generator, and micro gas turbine, and the insufficient part is supplemented by the power grid [16]. The electric energy storage device plays a peak-regulating role. The heat energy generated by the microturbine is used by the waste heat boiler for joint heating with the gas boiler, and the excess heat is stored as heat energy storage. The cooling load is supplied by absorption refrigerating machine and electric refrigerating machine.

\subsection{Mathematical Model of Photovoltaic Power Generation.} When there is no wind, the light intensity is $1000 \mathrm{~W} / \mathrm{m}^{2}$ and the temperature is $25^{\circ} \mathrm{C}$; the relationship among photovoltaic power generation power $[17,18]$, light intensity, and ambient temperature can be expressed as follows:

$$
P_{\mathrm{pv}}=f_{\mathrm{pv}} P_{\mathrm{STC}} \frac{G_{T}}{G_{\mathrm{STC}}}\left[1+k\left(T_{c}-T_{\mathrm{STC}}\right)\right],
$$

where $f_{\mathrm{pv}}$ is a photovoltaic array output power derating factor that takes into account the power loss of photovoltaic cells due to structural aging and surface covering. Under standard measurement environment, $P_{\mathrm{STC}}, G_{\mathrm{STC}}$, and $T_{\mathrm{STC}}$ are $\mathrm{PV}$ output peak power $(1000 \mathrm{Wp})$, light intensity $\left(1000 \mathrm{~W} / \mathrm{m}^{2}\right)$, and temperature $\left({ }^{\circ} \mathrm{C}\right)$, respectively. $G_{\mathrm{T}}$ is the actual light intensity of photovoltaic array; $k$ is the temperature coefficient of photovoltaic cell; and $T_{\mathrm{c}}$ is the actual temperature of photovoltaic cells, which is jointly determined by environmental temperature and light intensity [19]. The relationship can be expressed as follows:

$$
T_{c}=T_{\text {air }}+\frac{30 G_{T}}{1000},
$$

where $T_{\text {air }}$ represents the temperature and $G_{T}$ is the intensity of light radiation received by photovoltaic panels. From the above formula, it can be seen that when the light radiation intensity ranges from $0.4 \mathrm{~K} \cdot \mathrm{W} / \mathrm{m}^{2}$ to $1 \mathrm{k} \cdot \mathrm{W} / \mathrm{m}^{2}$, the actual output of photovoltaic array is linearly related to the light radiation intensity, and the photovoltaic output reaches the peak at $1 \mathrm{k} \cdot \mathrm{W} / \mathrm{m}^{2}$. When the light intensity is greater than $1 \mathrm{k} \mathrm{W} / \mathrm{m}^{2}$, the peak value will be maintained. When the light intensity is less than $0.4 \mathrm{k} \cdot \mathrm{W} / \mathrm{m}^{2}$, the power generation efficiency will decrease [20]. Based on this, the relationship between photovoltaic cell power generation and light intensity can be obtained as follows:

$$
P_{\mathrm{pv}}=\left\{\begin{array}{l}
\left(0.9+0.1 \frac{G_{T}}{0.4}\right) \times\left(G_{T} \times \frac{P_{\mathrm{NTC}}}{0.8}\right), G<0.4, \\
\left(G_{T} \times \frac{P_{\mathrm{NTC}}}{0.8}\right), 0.4<G<1, \\
\frac{P_{\mathrm{NTC}}}{0.8}, G>1,
\end{array}\right.
$$




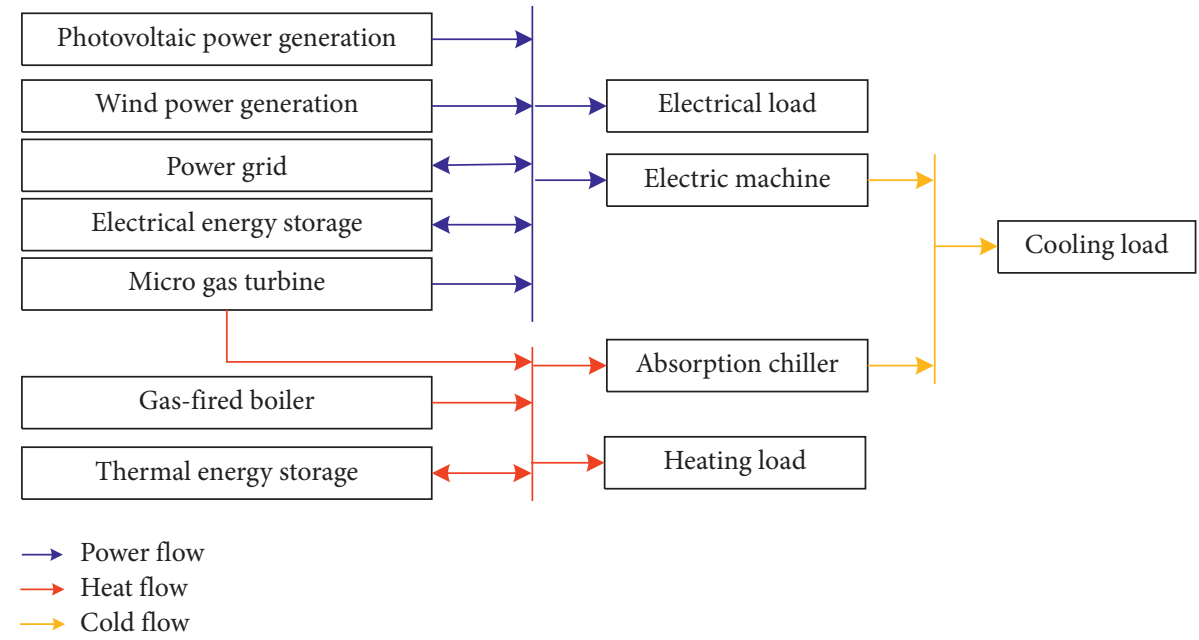

FIGURE 1: Schematic diagram of CCHP microgrid power supply structure.

where $P_{\mathrm{pv}}$ is photovoltaic power and $P_{\mathrm{NTC}}$ is the output power of photovoltaic under rated condition. The rated condition refers to the following: wind speed is $1 \mathrm{~m} / \mathrm{s}$, light radiation intensity is $800 \mathrm{~W} / \mathrm{m}^{2}$, and temperature is $20^{\circ} \mathrm{C}$.

2.3. Mathematical Model of Wind Power Generation. The wind turbine system is a new clean energy which uses wind turbine (WT) to convert wind energy into electric energy and supply it to users. It is one of the new clean energies with large application potential and fast development. The wind power generation system generally includes wind turbines, speed regulating devices, inverters, and controllers [21]. The fan output is affected by the change of wind speed, and the mathematical relationship between them can be expressed as follows:

$$
P_{\mathrm{wt}}=\frac{1}{2} \rho \pi R^{2} v^{3} C_{p}
$$

where $P_{\mathrm{wt}}$ is the generating power of wind turbine; $v$ is the actual wind speed; $\rho$ and $R$ are the air density $\left(\mathrm{kg} / \mathrm{m}^{3}\right)$ and fan wheel blade radius $(\mathrm{m})$, respectively; and $C_{p}$ is the wind energy conversion efficiency considering turbine loss and transmission loss.

The mathematical relationship between the actual output power and wind speed of a steady-state time-wind unit can be denoted as follows:

$$
P_{\mathrm{wt}}=\left\{\begin{array}{l}
0,0 \leq v \leq v_{\mathrm{in}}, \\
P_{r} \frac{v^{3}-v_{\text {in }}^{3}}{v_{r}^{3}-v_{\text {in }}^{3}}, v_{\text {in }} \leq v<v_{r}, \\
P_{r}, v_{r} \leq v<v_{\text {out }}, \\
0, v>v_{\text {out }},
\end{array}\right.
$$

where $P_{r}$ and $v_{r}$ are rated power and rated power of the fan, respectively, while $v, v_{\text {in }}$, and $v_{\text {out }}$ represent the actual wind speed of the fan, the input wind speed, and the output wind speed, respectively. The wind speed affects the fan's power which can be written as follows:

$$
v=v_{\text {ref }}\left(\frac{H}{H_{\text {ref }}}\right)^{\alpha} \text {, }
$$

where $H_{\text {ref }}$ and $v_{\text {ref }}$ are the height and wind speed of the measurement point, respectively; $H$ is the height of fan hub; and $\alpha$ is ground roughness factor.

2.4. Model of Pumped Storage Power Station. Pumped storage (PS), also known as pumped storage hydropower station, is essentially a device for storing and reusing water energy [22]. The pumped storage power generation system is a complex control system of water, machine, and electricity. It consists of a pressure water diversion system, inverter type unit, governor, and generator motor, among which reversible type unit has water pump and turbine.

When the water pump of an energy storage power station is operated under the pumping energy storage condition, the relationship between the converted power on the turbine and the storage capacity and energy storage can be expressed as follows:

$$
\left\{\begin{array}{l}
P_{\mathrm{ps}, \mathrm{ch}}=\left(\frac{\pi}{30}\right) M_{1} n_{1} D_{1}^{2} h^{1.5}, \\
E(t)=E(t-1)+P_{\mathrm{ps}, \mathrm{ch}} \cdot \eta_{d} \cdot \Delta t,
\end{array}\right.
$$

where $P_{\mathrm{ps}, \mathrm{ch}}$ is the accumulative power; $M_{1}, n_{1}$, and $D_{1}$ are unit shaft, unit speed, and runner diameter of pump turbine, respectively; $h$ is the turbine head; $E(t)$ represents the reservoir energy storage after time period $t ; \eta_{d}$ refers to the pumped storage power station pumping efficiency; and $\Delta t$ is the pumping time interval.

When the turbine of a storage power station operates under the condition of discharging water, the mathematical relationship between the power converted on the shaft and the reservoir energy storage can be written as follows: 


$$
\left\{\begin{array}{l}
P_{\mathrm{ps}, \mathrm{dis}}=9.18 \mathrm{QD}_{1}^{2} h^{1.5}, \\
E(t)=E(t-1)+P_{\mathrm{ps}, \mathrm{dis}} \cdot \frac{\Delta t}{\eta_{g}},
\end{array}\right.
$$

where $P_{\mathrm{ps} \text {,dis }}$ is the discharge power; $Q=v / t$ represents the flow velocity; $h$ is the head of the turbine; $E(t)$ represents the upper reservoir energy storage after time period $t ; \eta_{g}$ is the pumped storage power station water efficiency; and $\Delta t$ is the water power generation time interval.

2.5. The Equipment Model of the Cooling, Heating, and Power Supply System. The mathematical model for generation and heating of the micro gas turbine used in CCHP system can be calculated as follows:

$$
\begin{aligned}
P_{\mathrm{mt}} & =V_{\mathrm{mt}} \cdot H_{\mathrm{mt}} \cdot \eta_{\mathrm{mt}}, \\
\mathrm{Q}_{\mathrm{mt}} & =\frac{P_{\mathrm{mt}}\left(1-\eta_{\mathrm{mt}}-\eta_{\text {lost }}\right)}{\eta_{\mathrm{mt}}},
\end{aligned}
$$

where $P_{\mathrm{mt}}$ is the power output of the micro gas turbine; $Q_{\mathrm{mt}}$ is the residual heat of flue gas discharged simultaneously by the microturbine for power generation; $V_{\mathrm{mt}}$ represents the consumption of fossil fuels (mainly natural gas) per unit of time by the microturbine; $H_{\mathrm{mt}}$ refers to the low calorific value of natural gas, usually $9.780 \mathrm{Wh} / \mathrm{m}^{3}$; and $\eta_{\mathrm{mt}}$ and $\eta_{\text {lost }}$ are the micro gas turbine power generation efficiency and heat loss coefficient, respectively.

The output of an absorption chiller converting the heat input into heating or cooling can be expressed as follows:

$$
\begin{aligned}
& Q_{\text {cool }}=\operatorname{COP}_{\text {cool }} \cdot Q_{\text {in }}, \\
& Q_{\text {heat }}=\operatorname{COP}_{\text {heat }} \cdot Q_{\text {in }},
\end{aligned}
$$

where $Q_{\text {cool }}$ and $Q_{\text {heat }}$ are the cooling and heat generation power of waste heat absorption refrigerator, respectively; $\mathrm{COP}_{\mathrm{cool}}$ and $\mathrm{COP}_{\text {heat }}$ are the energy efficiency coefficient of refrigeration and heat energy efficiency of waste heat absorption refrigerator, respectively $\left(\mathrm{COP}_{\text {cool }}=0.9 ; \mathrm{COP}_{\text {heat }}=1.2\right)$; and $Q_{\text {in }}$ is the heat input power of waste heat absorption refrigerator.

The cooling power produced by the compression refrigerator and the electric energy consumed can be expressed as follows:

$$
\mathrm{Q}_{\mathrm{ec}}=\mathrm{COP}_{\mathrm{ec}} \cdot P_{\mathrm{ec}} \text {, }
$$

where $Q_{\mathrm{ec}}$ is the refrigeration power of compression refrigerator; $\mathrm{COP}_{\mathrm{ec}}$ represents the refrigeration efficiency coefficient, which is a factor to measure the refrigeration performance of the compression refrigerator; and $P_{\mathrm{ec}}$ is the power consumed by the compression refrigerator.

The relationship among the heating power of gas-fired boiler and its output and the amount of natural gas can be expressed as follows:

$$
Q_{\mathrm{gb}}=\eta_{\mathrm{gb}} \cdot F_{\mathrm{gb}}
$$

where $Q_{\mathrm{gb}}$ is the heating power of gas-fired boiler; $\eta_{\mathrm{gb}}$ is the gas boiler heating energy efficiency coefficient; and $F_{\mathrm{gb}}$ is the amount of fuel consumed by a gas-fired boiler.

The dynamic mathematical model of heat storage (HS) can be expressed as follows:

$$
E_{\mathrm{hs}}(t)=E_{\mathrm{hs}}(t-1) \cdot\left(1-\delta_{\mathrm{hs}}\right)+\left(Q_{\mathrm{hs}}^{\mathrm{ch}} \cdot \eta_{\mathrm{hs}}^{\mathrm{ch}}-\frac{\mathrm{Q}_{\mathrm{hs}}^{\mathrm{dis}}}{\eta_{\mathrm{hs}}^{\mathrm{dis}}}\right) \cdot \Delta t,
$$

where $E_{\mathrm{hs}}(t)$ represents the heat storage state of the regenerator at time $t ; Q_{\mathrm{hs}}^{\mathrm{ch}}$ and $Q_{\mathrm{hs}}^{\mathrm{dis}}$ represent the heat storage power and the heat release power, respectively; $\delta_{\mathrm{hs}}$ represents the self-release heat rate of the equipment; and $\eta_{\mathrm{hs}}^{\mathrm{ch}}$ and $\eta_{\mathrm{hs}}^{\mathrm{dis}}$ represent heat storage efficiency and heat release efficiency, respectively.

\section{Load Forecasting Based on PSO-KELM}

The key factors influencing the PV generation and load power were firstly studied to determine the input and output variables of the prediction model. Then, particle swarm optimization is used to optimize KELM parameters, and optimized models are used to predict photovoltaic output power and electric load power, respectively.

3.1. Influencing Factors of $P V$ and Load Forecasting. Figure 2 shows the relationship between photovoltaic output power and meteorological factors. The data in this paper are historical data collected from a microgrid photovoltaic power generation system from March to May 2015 in Hubei, China.

Figure 2(a) shows the variation of PV output power and light intensity curves. It can be seen from Figure 2(b) that temperature and PV output power have basically similar variation rules. It can be seen from Figure 2(c) that the PV output curve is basically opposite to the relative humidity curve. Figure 2(d) shows that there is no correlation between wind speed and PV output change curve. Through the above analysis, it can be known that solar radiation intensity, temperature, weather type, and relative humidity affect photovoltaic output power. Daily maximum light radiation intensity, daily maximum temperature, weather type index, daily maximum humidity, and historical PV output power containing PV system information are considered as the input and output variables of the prediction model.

Figure 3(a) shows the electricity load curve of an area for two consecutive weeks (April 13, 2015, solstice to April 26, 2015). Figure 3(b) shows the load change curve under different day types. Figure 3(c) shows the relationship between average daily load power and average daily temperature in a region. Figure $3(\mathrm{~d})$ shows the relationship between the average daily load power and humidity in a region. The data are historical data collected from a microgrid photovoltaic power generation system from March to May 2015 in Hubei, China.

It can be seen from Figure 3(a) that the load has a similar 
change rule within two weeks, which is reflected by a cycle of 7 days. It can be seen from Figure 3(b) that the load on working days is slightly higher than that on rest days, but the variation trend is similar, while the load on holidays is the lowest, which indicates that different types of days have different influences on load power. As can be seen from Figure 3(c), the average daily load presents an opposite trend to the average daily temperature and humidity.

Through the above analysis on the influencing factors of load prediction, it can be known that the main influencing factors of load power include historical load data, environmental temperature, humidity, and day type. Therefore, daily maximum temperature, daily minimum temperature, daily average temperature, daily type index, daily average humidity, and historical load power are taken as the input variables of the prediction model.

\subsection{Prediction Model Based on PSO-KELM}

3.2.1. The Mathematical Model of ELM. A new method of feedforward neural network training-extreme learning machine (ELM) - was proposed by H.G. in 2006 [23].

Compared with traditional backpropagation (BP), the weights and thresholds of hidden layer neurons are randomly given, which greatly improved calculation speed of ELM. The mathematical model of ELM is shown in Figure 4.

Training set $\left\{\left(x_{i}, t_{i}\right)\right\}_{i=1}^{N} \subset R^{n} \times R^{m}$, and excitation function $g(\cdot)$ of hidden node is a nonlinear function, which can be selected as Hard lim, Sigmoid, and Gaussian. The number of hidden layer neurons is $L$.

(1) Hidden layer parameters $\left(a_{i}, b_{i}\right) i=1, \ldots, L$ are randomly selected. $a_{i}$ is the input weight of the hidden layer neuron, and $b_{i}$ is the threshold value of the hidden layer neuron.

(2) The hidden layer output matrix $H=g\left(a_{i}, b_{i}, x_{i}\right)$ can be calculated as follows:

$H=\left[\begin{array}{c}h\left(x_{1}\right) \\ \vdots \\ h\left(x_{n}\right)\end{array}\right]=\left[\begin{array}{ccc}g\left(a_{1}, b_{1}, x_{1}\right) & \ldots & g\left(a_{L}, b_{L}, x_{1}\right) \\ \vdots & \cdots & \vdots \\ g\left(a_{1}, b_{1}, x_{n}\right) & \cdots & g\left(a_{L}, b_{L}, x_{n}\right)\end{array}\right]_{n \times L}$

(3) The output weight $\beta$ can be represented as follows:

$$
\beta=H^{+} T, \beta=\left[\begin{array}{c}
\beta_{1}^{T} \\
\vdots \\
\beta_{L}^{T}
\end{array}\right]_{L \times \mathrm{m}}, T=\left[\begin{array}{c}
t_{1}^{T} \\
\vdots \\
t_{n}^{T}
\end{array}\right]_{n \times \mathrm{m}}
$$

where $\mathrm{H}^{+}$is the left pseudoinverse matrix of the hidden layer output matrix $H$ and $T$ is the target output, that is, $T=\left\{t_{j}\right\}_{j=1}^{N}$.

(4) Calculate the output value $O_{j}$ by (16). Until the training error is less than the predetermined constant $\varepsilon$, these training samples can be approached by ELM.

$$
O_{j}=\sum_{i=1}^{L} \beta_{i} g\left(a_{i}, b_{i}, x_{i}\right),\left|O_{j}-T_{j}\right| \leq \varepsilon, \quad j=1, \ldots, n .
$$

(5) Error calculation:

$$
E_{\left(a_{i}, b_{i}\right)}=\frac{1}{n} \sum_{j=1}^{n}\left(O_{j}-T_{j}\right)^{2}
$$

where $\left(a_{i}, b_{i}\right)$ is the hidden layer node weight and threshold, $T_{j}$ is the predicted value of the $j$ th output node, and $O_{j}$ is the actual value of the $j$ th output node.

In order to further enhance the generalization ability and stability of ELM, the kernel function is introduced into ELM by comparing the principle of ELM and support vector machine (SVM), and the KELM algorithm is proposed.

(1) The kernel matrix is defined by Mercer's conditions as shown in the following equation:

$$
\left\{\begin{array}{l}
\Omega_{\mathrm{ELM}}=H H^{T} \\
\Omega_{i, j}=h\left(x_{j}\right) \cdot h\left(x_{j}\right)=K\left(x_{i}, x_{j}\right) .
\end{array}\right.
$$

The random matrix $H H^{T}$ of ELM is replaced by nuclear matrix $\Omega$. Using the kernel, all input samples are mapped from the $N$-dimensional input space to the high-dimensional hidden layer feature space. When the kernel parameters in $\Omega$ are determined, the value of $\Omega$ can be obtained. $h(x)$ is the output function of hidden layer node. Kernel $K(\mu, v)$ is usually set as follows:

$$
K(\mu, v)=\exp \left[-\left(\mu-\frac{v^{2}}{\sigma}\right)\right]
$$

(2) I/C is added to the main diagonal of the unit diagonal matrix $H H^{T}$ so that its characteristic root is not zero, and then the weight vector $\beta^{*}$ is evaluated as follows:

$$
\beta^{*}=H^{T}\left(\frac{I}{C}+H H^{T}\right)^{-1} T \text {. }
$$

The output of the KELM model can be expressed as follows:

$$
\begin{aligned}
f(x) & =h(x) H^{T}\left(\frac{I}{C}+H H^{T}\right)^{-1} T \\
& =\left[\begin{array}{c}
K\left(x, x_{1}\right) \\
\cdots \\
K\left(x, x_{N}\right)
\end{array}\right]\left(\frac{I}{C}+\Omega_{\mathrm{ELM}}\right)^{-1} T .
\end{aligned}
$$

The KELM output weight can be expressed as follows: 


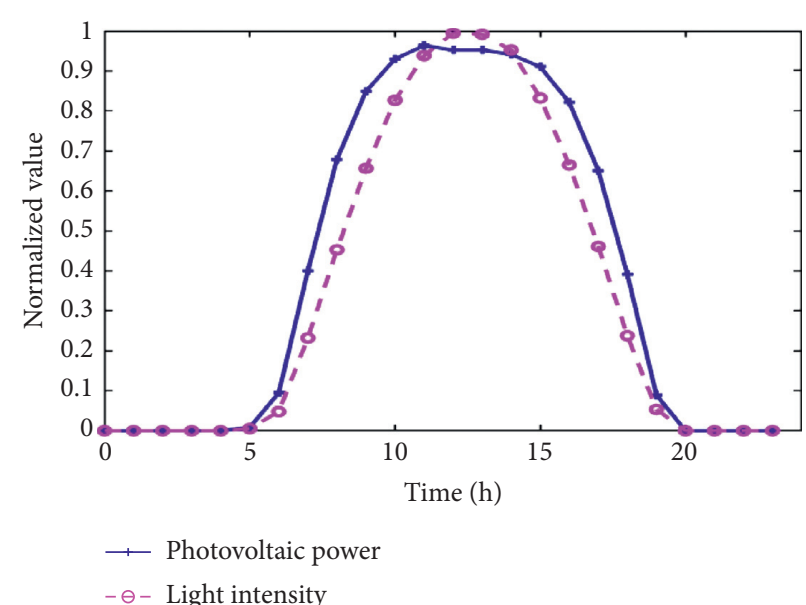

(a)

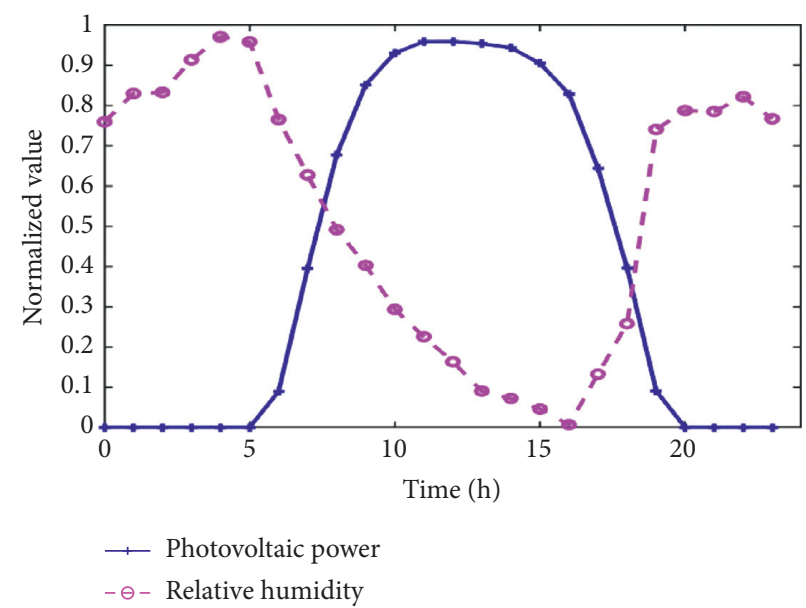

(c)

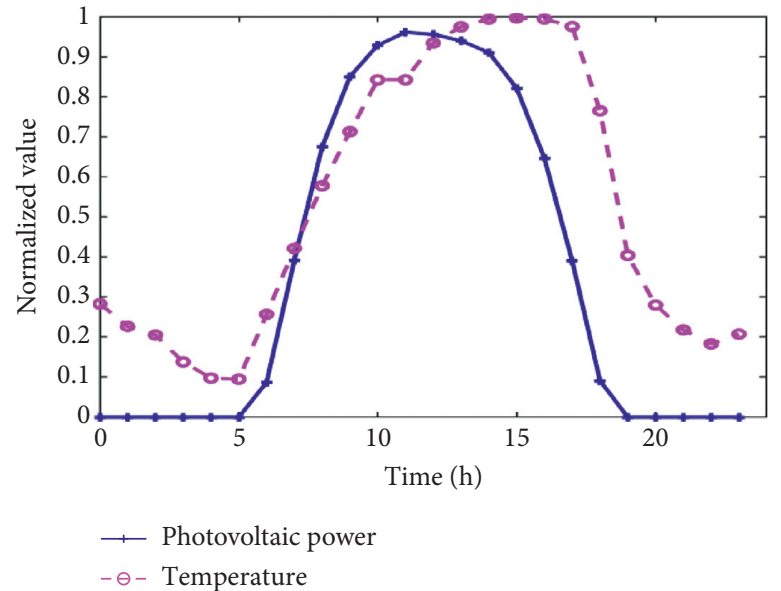

(b)

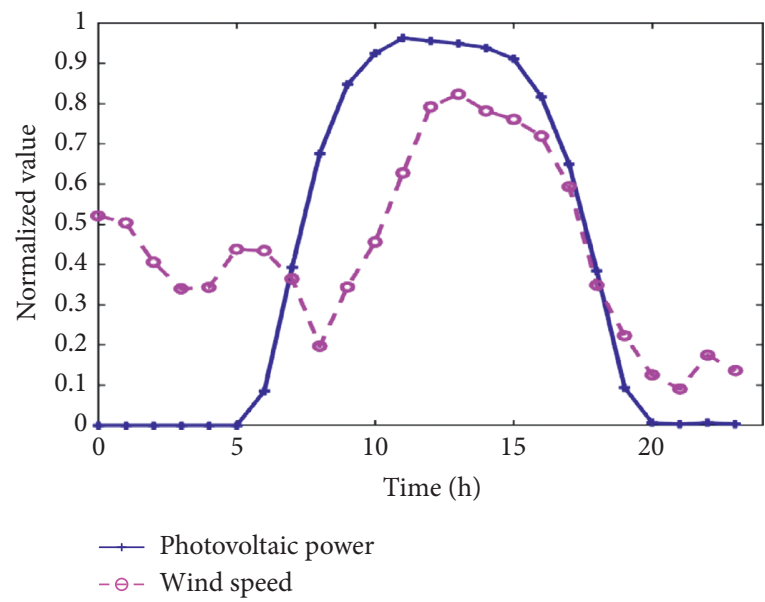

(d)

FIGURE 2: Relationship between photovoltaic power generation and meteorology. (a) Relationship diagram of PV and light intensity. (b) Relationship diagram of PV and temperature. (c) Relationship of PV and relative humidity. (d) Relationship of PV and wind speed.

$$
\beta=\left(\frac{I}{C}+\Omega_{\mathrm{ELM}}\right)^{-1} T
$$

3.2.2. The Mathematical Model of PSO. There are $n$ particles in $m$-dimensional space. The position of the $i$ th particle in $m$ dimensional space can be expressed as $x_{i}\left(x_{i 1}, x_{i 2}, \cdots x_{\text {im }}\right)$ [19].
The best position experienced by the $i$ th particle is recorded as $p_{i}\left(p_{i 2}, p_{i 2}, \cdots, p_{\text {im }}\right)$. The velocity of each particle is defined as $v_{i}\left(v_{i 1}, v_{i 2}, \cdots, v_{\mathrm{im}}\right)$ [24]. The best place for all particles to pass is written as $p_{g}\left(p_{i 1}, p_{i 2}, \cdots, p_{\text {im }}\right)$ [25].

After the optimal solution is determined, the particle velocity and position can be updated according to (23)-(25).

$$
\begin{aligned}
v_{\mathrm{im}}^{k}(t+1) & =\omega v_{\mathrm{im}}^{k}(t)+c_{1} r_{1}\left(\mathrm{pbest}_{\mathrm{im}}^{k}-x_{\mathrm{im}}^{k}(t)\right)+c_{2} r_{2}\left(\text { gbest }_{\mathrm{im}}^{k}-x_{\mathrm{im}}^{k}(t)\right), \\
x_{\mathrm{im}}^{k}(t+1) & =x_{\mathrm{im}}^{k}(t)+v_{\mathrm{im}}^{k}(t+1), \\
\omega & =\omega_{\max }-k \frac{\left(\omega_{\max }-\omega_{\min }\right)}{k_{\max }},
\end{aligned}
$$

where $v_{\mathrm{im}}^{k}$ is the velocity of particle $i$ in the $k$ th iteration; $x_{\mathrm{im}}^{k}$ is the position of particle $i$ in the $k$ th iteration; $k$ is the current evolutionary algebra; $k_{\max }$ is the maximum evolutionary algebra; $\omega$ is the weight of inertia; $i=1,2,3, \cdots, M$, is the population size; $c_{1}$ and $c_{2}$ are learning factors; $r_{1}$ and $r_{2}$ are random numbers between $[0,1]$; pbest ${ }_{i m}^{k}$ is the position of 


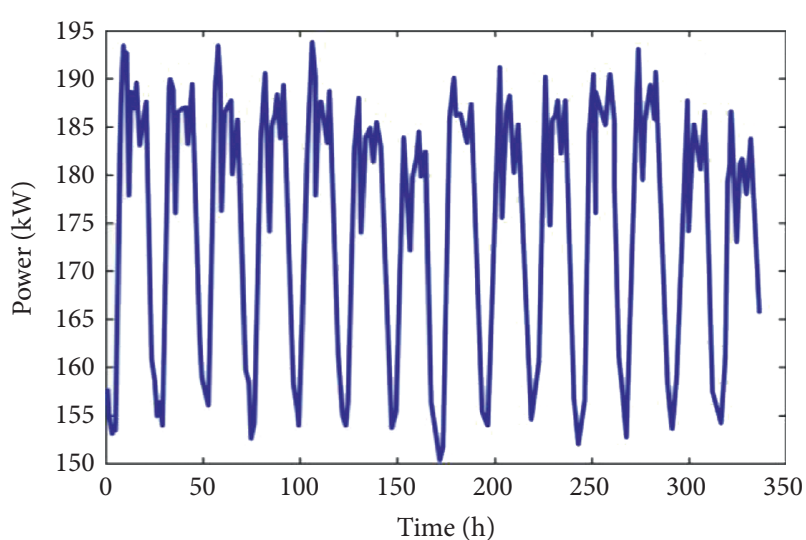

— Load

(a)

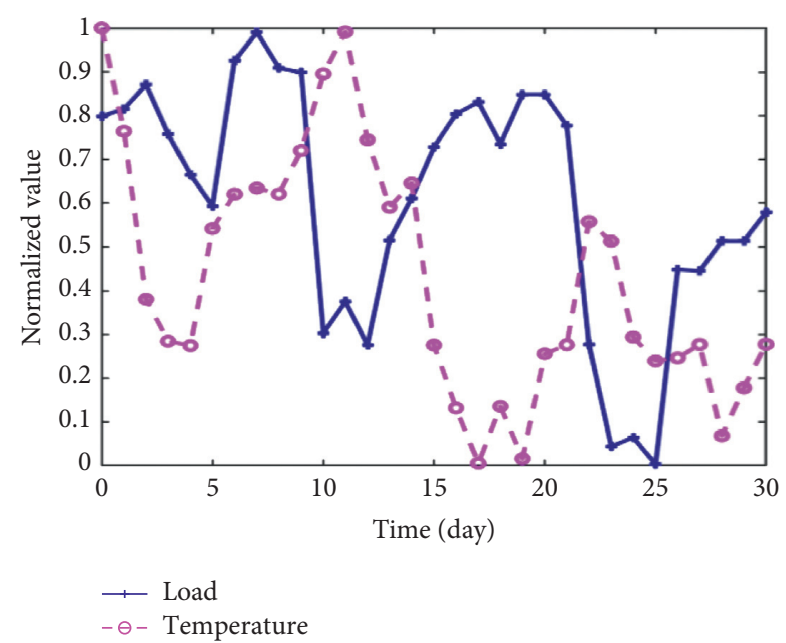

(c)

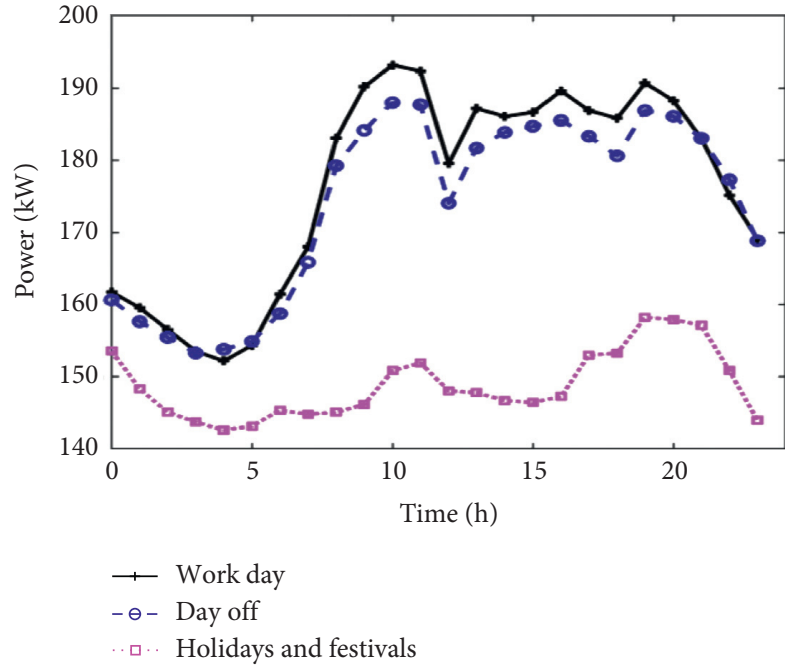

(b)

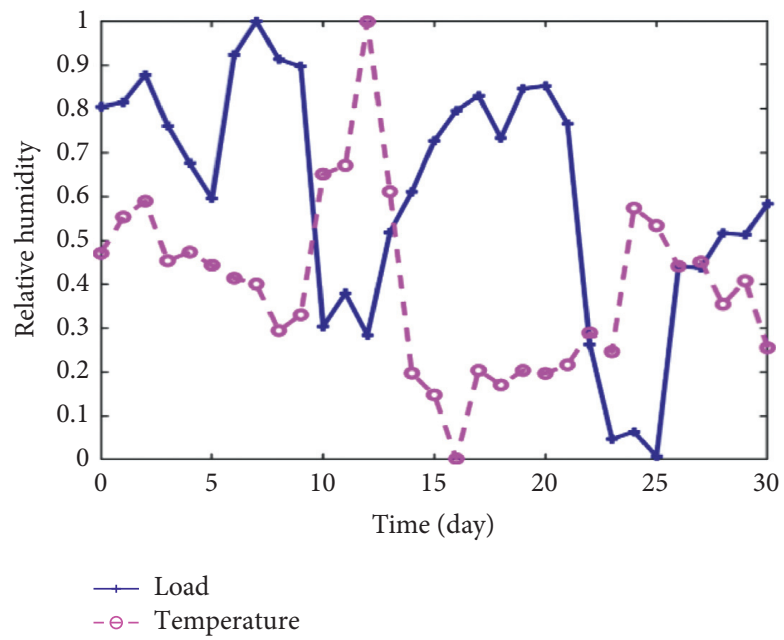

(d)

FIGURE 3: Influencing factors of load power. (a) Two-week electrical load graph. (b) Load change curve under different day types. (c) Relationship between load and temperature. (d) Relationship between load and temperature.

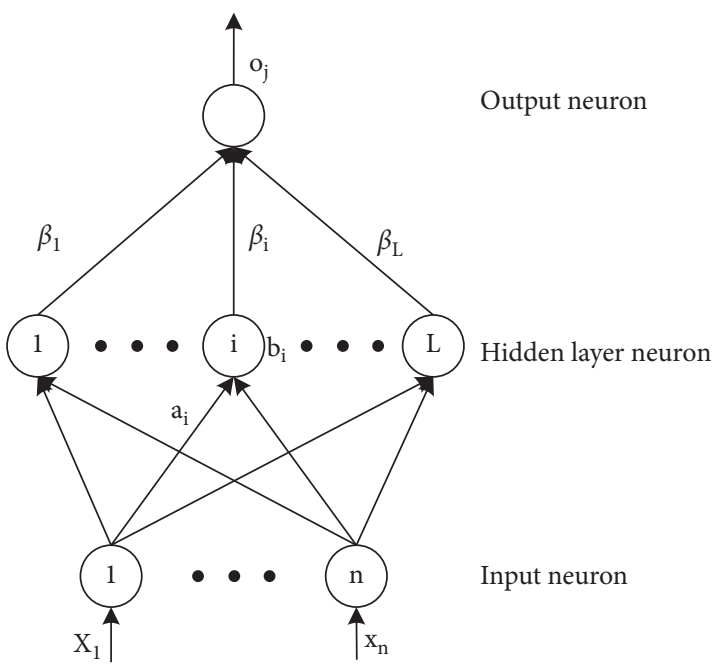

FIgURE 4: The structure of ELM. the individual extremum point of particle $i$; and $g b e s t_{\mathrm{im}}^{k}$ is the extremum of the whole population. Each particle's speed is limited to $\left[-v_{\max },+v_{\max }\right]$.

The implementation of particle swarm optimization can be described as follows [26-29].

Step 1: initialize particle swarm.

Population size $N$, initial position and velocity vector of each particle, individual extreme value and global optimal solution, iteration error accuracy $\varepsilon$, constant coefficients $c_{1}$ and $c_{2}$, maximum and minimum inertial values $\omega_{\max }$ and $\omega_{\min }$, maximum velocity $v_{\max }$, and maximum number of iterations $T_{\max }$ are set.

Step 2: particalization of the solution of the target problem. The solution of the target problem to be solved is described by the position vector of the particles, and the fitness function of the particles in the particle swarm is determined. 
Step 3: the individual extremum of each particle and the global extremum of the population are calculated.

Step 4: particle velocity and position are updated.

Step 5: determine whether the following conditions are satisfied. If so, turn to Step 6; otherwise, turn to Step 2.

The iteration error reaches the set precision.

The iteration number of the algorithm reaches the preset maximum iteration number Tmax.

Step 6: output the optimal solution. The particle global optimal value and its corresponding position in the particle swarm are converted into the optimal value and corresponding solution of the target problem, and the algorithm ends.

The flowchart of PSO-KELM algorithm is shown in Figure 5.

The regularization coefficient $\mathrm{C}$ and the nuclear parameter are optimized by PSO, which have great influence on the accuracy of the prediction model of KELM, and then the wind power prediction model is established by PSOKELM.

The PSO-KELM algorithm flowchart can be represented as follows:

$$
\text { fitness }=\frac{1}{n} \sum_{i=1}^{n}\left|\frac{y_{p, i}-y_{t, i}}{y_{t, i}}\right| \text {, }
$$

where $y_{p, i}$ is the original data, $y_{t, i}$ is the predicted value, and fitness is the fitness function which reflects the error of model prediction.

The regularization coefficient and kernel parameters of KELM are optimized by PSO to avoid blind training of KELM. In the PSO-KELM prediction model, the output of the KELM learning sample and the root mean square error of the actual output are used as the fitness function of PSO, the fitness value of the particle is compared with the optimal fitness, and the parameters $C$ and $\lambda$ of each KELM are obtained.

The mean relative error is selected as the fitness function of algorithm optimization.

The process of photovoltaic and load forecasting model by PSO-KELM can be expressed as follows.

Step 1: the data affecting photovoltaic power generation or load prediction are divided into training set and test set, and the data are normalized.

Step 2: the kernel function of KELM is determined, RBF kernel is selected, and mapping input vector $\mathrm{Xi}$ and initial output weight $\beta_{\text {int }}$ are obtained.

Step 3: initialize particle swarm parameters, including population number, the initial position of the particle and random initial velocity, individual extremum, and total extremum.

Step 4: particle swarm optimization. According to the objective function, the fitness of each particle is calculated at each iteration, and the speed, position, and global optimal value are updated. The optimal output weight is obtained after iteration.

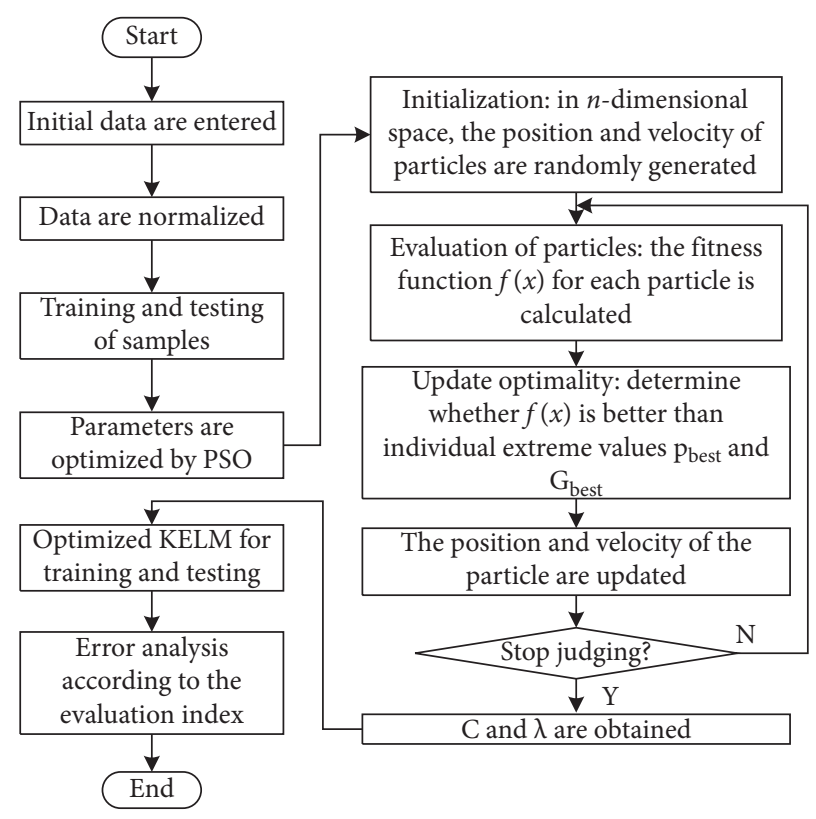

Figure 5: Flowchart of PSO-KELM algorithm.

Step 5: calculate the test data output according to the optimal KELM output weight, and the prediction results are obtained according to the evaluation index.

\section{CCHP Microgrid Scheduling Model Based on MSFLA}

\subsection{Problem Description}

4.1.1. Operating Cost. The optimal objective function of CCHP is the integrated minimum operation cost, which can be expressed as follows:

$$
\min f=f_{\text {om }}+f_{\text {grid }}+f_{\text {fuel }}+f_{\text {open }},
$$

where $f_{\text {om }}$ is the cost of operation and maintenance; $f_{\text {fuel }}$ is the cost of fuel consumption; $f_{\text {grid }}$ is the cost of power exchange with the grid (the marginal cost can be reduced by transferring power generation from high-cost units to lowcost units); and $f_{\text {open }}$ is the start-up and shut-down, and unit maintenance cost, equipment service life, and coal, oil, natural gas, and other costs are considered as downtime costs.

(1) Operation and maintenance cost can be expressed as follows:

$$
f_{\mathrm{om}}(t)=\sum_{i=1}^{N} K_{\mathrm{om}, i} \cdot P_{i}(t)
$$

where $K_{\mathrm{om}, i}$ and $P_{i}$ represent the maintenance coefficient and output size $(\mathrm{kW})$ of the $i$ th microsource, respectively.

(2) Fuel cost can be expressed as follows: 


$$
f_{\text {fuel }}(t)=C_{\mathrm{ng}} V=C_{\mathrm{ng}} \sum_{i=1}^{N} \frac{1}{\mathrm{LHV}} \frac{P_{i}(t)}{\eta_{i}},
$$

where $V$ represents the amount of natural gas consumed $\left(\mathrm{m}^{3}\right), C_{\mathrm{ng}}$ refers to the price of natural gas $\left(\mathrm{CNY} / \mathrm{m}^{3}\right), \mathrm{LHV}$ represents the low calorific value of natural gas, and $\eta_{i}$ and $P_{i}(t)$ represent the output of the $i$ th microsource $(\mathrm{kW})$ and the corresponding generation efficiency, respectively.

(4) Electric power exchange cost can be written as follows:

$$
f_{g}(t)=\left\{\begin{array}{l}
C_{b}(t) \cdot P_{\text {grid }}(t), P_{\text {grid }}(t)>0 \\
C_{s}(t) \cdot P_{\text {grid }}(t), P_{\text {grid }}(t)<0
\end{array},\right.
$$

where $C_{b}(t)$ and $C_{s}(t)$ are the purchase price and sale price $(\mathrm{CNY} / \mathrm{kW})$ of the power grid at time $T$, respectively, and $P_{\text {grid }}(t)$ is the interaction power $(\mathrm{kW})$ with the grid at time $t$. Positive value means the power purchased from the side of the power grid by the microgrid, while negative value means the power sold by the microgrid.

(5) Downtime cost can be defined as follows:

$$
f_{\text {open }}(t)=\sum_{i=1}^{N} \sigma_{i}(t) \cdot\left(1-\sigma_{i}(t-1)\right) \cdot C_{i, \text { open }},
$$

where $\sigma_{i}(t)$ and $\sigma_{i}(t-1)$ are the switching state of the $i$ th microsource (microturbine or gas boiler) at the current moment and the previous moment, respectively; 1 means turn on; 0 means turn off; and $C_{i, \text { open }}$ represents the start-up cost $(\mathrm{CNY})$ of the $i$ th microsource.

4.1.2. Constraints. Load balance constraint and operation characteristic constraint of each microsource are the main constraints considered in microgrid.

(1) Electric load balance can be defined as follows:

$P_{\mathrm{Lnet}}(t)+P_{\mathrm{ec}}(t)=P_{\mathrm{mt}}(t)+P_{\mathrm{ps}}(t)+P_{\text {grid }}(t)$,

where $P_{\text {Lnet }}(t), P_{\mathrm{ec}}(t), P_{\mathrm{mt}}(t), P_{\mathrm{ps}}(t)$, and $P_{\text {grid }}(t)$ are net electric load, microturbine output, pumped storage output, electric refrigerator power consumption, and power exchange with large power grid at time $t$, respectively. The net electric load is the difference between the total electric load and the wind-solar power, that is, $P_{\text {Lnet }}(t)=P_{L}(t)-P_{\mathrm{WT}}(t)-P_{\mathrm{PV}}(t)$.

(2) Cooling load balance can be expressed as follows:

$$
Q_{\mathrm{ac}, \text { cool }}(t)+Q_{\mathrm{et}}(t)=Q_{c}(t)
$$

where $Q_{c}(t)$ is the cooling load at time $t$ and $Q_{\mathrm{ac}, \text { cool }}(t)$ and $\mathrm{Q}_{\mathrm{et}}(t)$ are the cooling power of absorption refrigerator and electric refrigerator at time $t$, respectively.

(3) Thermal load balance can be expressed as follows:

$Q_{\mathrm{mt}}(t) \eta_{\mathrm{rec}}+Q_{\mathrm{ac}, \text { in }}(t)+Q_{\mathrm{gb}}(t)+Q_{\mathrm{hs}}(t)=Q_{h}(t)$,

where $Q_{\mathrm{mt}}(t), Q_{\mathrm{ac}, \text { in }}(t)$, and $Q_{\mathrm{gb}}(t)$ are the flue gas waste heat power generated by the micro gas turbine machine, the heat consumption power of the absorption refrigerator, and the heat generation power of the gas boiler at time $t . Q_{\mathrm{hs}}(t)$ is the heat storage and release power of the heat storage device at time $t$. The heat release is positive and the heat storage is negative. $Q_{h}(t)$ is the thermal load at time $t . \eta_{\mathrm{rec}}$ is the conversion efficiency of waste heat recovery unit.

(4) Equipment output constraint can be expressed as follows:

$$
P_{\min , i} \leq P_{i}(t) \leq P_{\max , i}
$$

where $P_{\min , i}$ and $P_{\max , i}$ represent the output upper and lower limits of equipment unit $i$, respectively.

(5) Grid interaction power constraint can be written as follows:

$$
P_{\text {grid }}^{\min } \leq P_{\text {grid }} \leq P_{\text {grid }}^{\max }
$$

where $P_{\text {grid }}^{\min }$ and $P_{\text {grid }}^{\max }$ refer to the upper and lower limits of the maximum exchange power between the microgrid system and the large grid, respectively.

(6) Equipment climbing constraints can be expressed as follows:

$$
\left\{\begin{array}{l}
P_{i}(t)-P_{i}(t-1) \leq P_{i}^{\text {up }}, \\
P_{i}(t-1)-P_{i}(t) \leq P_{i}^{\text {down }} .
\end{array}\right.
$$

(7) Initial and final balance constraint of energy storage.

Cycle continuity of optimized operation of microgrid system and time of energy storage unit (pumped storage and heat storage tank).

When segment coupling is considered, the initial and final energy storage of multiperiod periodic dispatch needs to be consistent. It can be described as follows:

$$
E_{0}=E_{\text {end }} \text {, }
$$


where $E_{0}$ and $E_{\text {end }}$ are energy storage at the beginning and end of cycle dispatch of energy storage devices, respectively.

4.1.3. Constraint Processing Method. The inequality constraints of climbing constraints and output upper and lower limits are combined as follows:

$$
\max \left(P_{\min , i}, P_{i}(t-1)-P_{i}^{\mathrm{down}}\right) \leq P_{i}(t) \leq \min \left(P_{\max , i}, P_{i}(t-1)+P_{i}^{\mathrm{up}}\right) .
$$

The equipment output update needs to be satisfied with equation (40). When the constraint is not satisfied, the formula is adjusted as follows:

$$
P_{i}(t)=\left\{\begin{array}{l}
\max \left(P_{\text {min }, i}, P_{i}(t-1)-P_{i}^{\mathrm{down}}\right), P_{i}(t)<\max \left(P_{\min , i}, P_{i}(t-1)-P_{i}^{\mathrm{down}}\right) \\
\min \left(P_{\mathrm{max}, i}, P_{i}(t-1)+P_{i}^{\mathrm{up}}\right), P_{i}(t)>\min \left(P_{\mathrm{max}, i}, P_{i}(t-1)+P_{i}^{\mathrm{up}}\right)
\end{array},\right.
$$

where $P_{i}(t)$ and $P_{i}(t-1)$ represent the outputs of the $i$ th equipment unit at the current moment and the previous moment, respectively, and $P_{i}^{\text {up }}$ and $P_{i}^{\text {down }}$ represent the maximum increased power output and maximum decreased power output of equipment unit $i$ in time period $t$. Micro gas turbine and gas boiler are mainly considered as equipment with climbing constraints in this paper.

For the equipment without climbing constraint, but constrained by the output upper and lower limits, the formula is adjusted as follows:

$$
P_{i}(t)=\left\{\begin{array}{l}
P_{\min , i}, P_{i}(t)<P_{\min , i} \\
P_{i}(t), P_{\min }<P_{i}(t)<P_{\max , i} \\
P_{\max , i}, P_{i}(t)<P_{\max , i}
\end{array}\right.
$$

Considering the new objective function of energy storage constraints, take pumped storage power station as an example. It can be expressed as follows:

$$
\min f_{\text {new }}=f+\beta\left|E_{0}-E_{\text {end }}\right| .
$$

After the initial and final balance of the reservoir energy storage state in a pumped storage station is considered, the above equation can be modified as follows:

$$
\min f_{\text {new }}=f+\mu\left|\sum_{t=1}^{T} P_{d, t} \cdot \eta_{d}-\sum_{t=1}^{T} \frac{P_{g, t}}{\eta_{g}}\right|,
$$

where $P_{d, t}$ and $P_{g, t}$ are the storage discharge power of pumped storage power station in time period $t$ and $\eta_{d}$ and $\eta_{g}$ represent the storage discharge efficiencies of pumped storage station, respectively.

4.2. Shuffled Frog Leaping Algorithm. Inspired by shuffled frog group leaping and foraging behavior, a new shuffled frog algorithm (SFLA) was proposed. SFLA's mathematical model can be described as follows.

Assuming that the problem to be solved is a $D$ dimensional vector, the initial $P$ frogs are randomly generated within the feasible threshold. The location of the $i$ th frog can be represented as $x_{i}=\left\{x_{i 1}, x_{i 2}, \ldots, x_{i D}\right\}$, the fitness function value of each individual is calculated and arranged in descending order according to size, the optimal fitness value of the current population is selected, and the corresponding individual $x_{g}$ is recorded. Then, the population is divided into $M$ subpopulation groups, each subpopulation contains $N$ individuals, that is, $P=M \times N$, and the rules of division is that the first frog is assigned to the first subgroup and the second is assigned to the second subgroup. The $M$ frog is assigned to the $M$ subgroup, the $M+1$ frog is assigned to the first subgroup, and so on until all the individuals were divided.

Local search: the individuals with the best and worst adaptive values of each subpopulation are denoted as $x_{b}$ and $x_{w}$, respectively. At each iteration of the loop, the worst individual is updated in position. The update policy formula can be expressed as follows:

$$
\left\{\begin{array}{l}
D_{i}(t+1)=\operatorname{rand}() \cdot\left(x_{b}-x_{w}\right) \\
x_{w}(t+1)=x_{w}(t)+D_{i}(t+1)
\end{array}\right.
$$

where $D_{i} \in\left[D_{\min }, D_{\max }\right]$ is the update step size for each jump, $\operatorname{rand}()$ is the randomly generated number within the range of $[0,1]$, and $x_{w}(t+1)$ is the updated position of the individual.

Global search: after a fixed number of local searches are completed, all subpopulations are remixed into a single population so that the various group information can be communicated to each other. In descending order of fitness, the subgroups are regrouped, and local searches continue until the termination condition is met.

4.2.1. Flow of SFLA. The implementation process of SFLA is generally divided into four steps: initialization, population grouping, local search within cluster group, and cluster group remixing. The specific process can be described as follows [23]:

(1) Relevant parameters to initialize the population are input.

(2) Sorting: according to the characteristics of solving the problem, the fitness value of each frog in the population was calculated, and all the individuals were sorted according to the fitness value.

(3) Grouping: the population was divided into $M$ subgroups according to the fitness value. Each subgroup contained $N$ frogs. The optimal individual and the worst individual in each subgroup were recorded, respectively. 
(4) Subgroup local search: the worst individual in the subgroup is updated according to formula (44), and the subgroup and the global optimal individual are also updated.

(5) Judge whether the local search of subgroup reaches the maximum number of iterations; if not, jump to (4) to continue execution.

(6) Subgroup remixing: all subpopulations are mixed into one population, all individuals are arranged according to fitness value, and the global optimal individual information is updated.

(7) Check whether the global maximum number of evolutionary iterations or convergence accuracy meets the requirements; if so, exit the algorithm; otherwise, return back to (3).

\subsubsection{Improved Shuffled Frog Leaping Algorithm (MSFLA)}

(1) Cauchy mutation operator.

Cauchy distribution is a kind of functional distribution commonly used in mathematical statistics and other fields. Its probability density distribution function can be written as follows [24-26]:

$$
f(x)=\frac{\beta}{\pi\left(\beta^{2}+(x-\alpha)^{2}\right)},-\infty \leq x \leq \infty,
$$

where when $\alpha=0, \beta=1$ is satisfied, it is called the standard Cauchy distribution, denoted by $C(0,1)$.

When the traditional SFLA is iterated for many times, new individuals in the subgroup may fall into premature convergence. The variation of random numbers obeying Cauchy distribution will produce large update step, which is helpful for the population to jump out of local extremum. In this way, the optimization performance in the larger solution space is better and the global searching ability of the algorithm is improved. The improved local update strategy can be written as follows:

$$
\left\{\begin{array}{l}
D_{i}(t+1)=\operatorname{rand}() \cdot\left(x_{b}-x_{w}\right) \cdot C(0,1) \\
x_{w}(t+1)=x_{w}(t)+D_{i}(t+1)
\end{array}\right.
$$

(2) Adaptive variation.
The idea of adaptive mutation is introduced into SFLA and can be expressed as follows:

$$
p_{m}=\left\{\begin{array}{l}
k_{2}-\frac{\left(k_{2}-k_{1}\right) \cdot\left(f_{\mathrm{avg}}-f\left(x_{i}\right)\right)}{f_{\max }-f_{\mathrm{avg}}}, f\left(x_{i}\right)<f_{\mathrm{avg}} \\
k_{2}, f\left(x_{i}\right)>f_{\mathrm{avg}}
\end{array}\right.
$$

When the fitness value of an individual is better than the average fitness value, a lower mutation probability is assigned to protect the individual to enter the next iteration. On the contrary, if the fitness value of an individual is less than the average fitness value, the corresponding mutation probability is higher, and the individual can be eliminated.

(3) Disturbance operation.

If the diversity of the population is guaranteed, the global search capability is improved, and the individual updates are followed by another disturbance operation, which can be expressed as follows:

$$
\left\{\begin{array}{l}
r=\frac{\max \left(x_{j}\right)-\min \left(x_{j}\right)}{2}, \\
x_{i, j}(t)=x_{i, j}(t)+2 \cdot r \cdot \operatorname{rand}()-r,
\end{array}\right.
$$

where $r$ is the disturbance radius; $x_{j}$ is the $j$ th dimension value of the population individual; and $x_{i, j}$ is the $j$ th dimension value of the $i$ th individual of the population.

4.3. MSFLA-Based CCHP Microgrid Scheduling Model. With the day-ahead economic minimization cost of CCHP as the objective function, the scheduling model was solved by MSFLA. The steps for optimal operation of the microgrid can be expressed as follows.

(1) Input data are read and initialized. It mainly includes the number of the micropower supply kinds and the output restrictions of the microgrid system, the load demand, the data of wind power and photovoltaic power generation data, the initial parameters of every unit (pumped storage reservoir, microgas turbine, and thermal energy), the energy price information, equipment performance, the scheduling number $T$, and the parameters of MSFLA. 
(2) The population is randomly initialized. According to the output limitation of each microsource equipment, microturbine, pumped storage, heat storage tank, and gas boiler are selected as decision variables, and the initial population individuals are randomly generated in the feasible region, and their positions are represented as a group of feasible scheduling plans. The output of the electric refrigerator, the output of the absorption refrigerator, and the heat recovery power of waste heat can be determined by the balance constraint of the cold and heat power and the calculation of the decision variables. The interaction power with the grid can be calculated by load balancing constraints and decision variables. Fuel consumption, gas waste heat power of micro gas turbine and power consumption of refrigeration equipment can be calculated according to corresponding mathematical model and energy conversion coefficient.

(3) Individuals of the population are modified by constraints. The individuals in the population who violate the constraints are adjusted, the decision variables are adjusted back to the feasible solution space, and the adjusted population is obtained.

Equation (30) is used as the fitness function of algorithm optimization. Fitness values were calculated for each individual, and then all individuals were ranked in descending order according to fitness values.

(5) According to the fitness value, the population was divided into $M$ groups, each group containing $\mathrm{N}$ frogs, and the optimal and worst individuals in each subgroup were recorded, respectively.

(6) The worst individual in each subgroup is updated according to equation (47). According to formula (44), individuals are subjected to mutation operations. If the fitness value of the individual after variation is better than before, the replacement is carried out. Simultaneously, the clipboard is updated (subgroup and globally optimal individual).

(7) Judge whether subgroup search reaches the maximum iteration number of subgroups; if not, jump to (6) to continue execution.

(8) All subpopulations are mixed into one population, and all individuals are rearranged according to fitness value to update the global optimal individual information.

(9) Check whether the global maximum number of evolutionary iterations or convergence accuracy meets the requirements, and if so, jump to the next step. Otherwise, skip step (5).

(10) Global optimal value and corresponding decision variable value are output, output of each microsource equipment is solved, and optimal scheduling scheme is obtained.

\section{Simulation}

5.1. Photovoltaic Power Generation Forecast. The radial basis function (RBF) is selected, and set $\sigma=0.1$ and $C=0.5$. The number of PSO population is set as 25 , the initial output weight calculated by KELM is set as the initial position of the particle, the initial velocity of the particle is randomly selected in $[0,1]$, and the particle dimension is the output weight dimension. Table 1 shows the evaluation indexes of the predicted results under different weather conditions including KELM, GA-KELM, and PSO-KELM.

Figure 6 shows the comparison curve between the predicted value and the real value of photovoltaic output power under different prediction models in sunny days. In order to show the photovoltaic power generation prediction results of the three models, the relative error comparison between the predicted value and the true value is shown in Figure 7.

It can be seen from Figure 6 that the photovoltaic prediction results of the three models are generally consistent with the actual values. However, the PV prediction curve of GA-KELM in sunny days is superior to that of traditional KELM. In the three models, the predicted value of PSO-KELM is closer to the true value. It can be seen from Figure 7 that the prediction error of PSO-KELM is less than GA-KELM and KELM.

5.2. Power Prediction of Electric Load. The historical electric load power, the corresponding meteorological information, and the day type, calculated from March to May 2015, are selected as the training and prediction model. The electric load power is selected from 0:00 to 23:00, and the time interval is $1 \mathrm{~h}$. According to the above analysis of affecting factors of load forecasting, load power, the day before the same time, the day before the highest temperature, the lowest average temperature, average temperature, humidity, type index, two days before the load power at the same time, predicted daily maximum temperature, minimum temperature, average temperature, average humidity, and day type index are used as the prediction model of input. The output variable is the load power at the corresponding time of the predicted day.

Figure 8(a) shows the prediction results of KELM, GAKELM, and PSO-KELM on the working day. Figure 8(b) shows the relative error curves of the three prediction models for the load power prediction results.

Figures 9(a) and 9(b) show the prediction results of KELM, GA-KELM, and PSO-KELM on holidays.

As can be seen from Figures 8(a), 9(a), and 9(b), PSOKELM load forecasting results are more accurate than GAKELM and KELM, improving the global CCHP search capability. The reliability of the proposed method is verified. As can be seen from Figure 8(b), PSO-KELM prediction method has the smallest relative error at most times of a day, while KELM prediction model has the largest relative error. Therefore, PSO-KELM is a relatively better prediction model, with smaller relative error and higher accuracy. 
TABLE 1: Models' prediction and evaluation of different weather conditions.

\begin{tabular}{lcccccc}
\hline Prediction & \multicolumn{2}{c}{ Sunny } & \multicolumn{2}{c}{ Cloudy } & \multicolumn{2}{c}{ Rainy } \\
algorithm & RMSE & MAPE & RMSE & MAPE & RMSE & MAPE \\
\hline KELM & 0.17631 & 0.1011 & 0.60119 & 0.3328 & 0.29756 & 4.2544 \\
GA-KELM & 0.16517 & 0.0535 & 0.52726 & 0.1822 & 0.29564 & 3.7162 \\
PSO-KELM & 0.16376 & 0.0376 & 0.48986 & 0.1649 & 0.24274 & 3.714 \\
\hline
\end{tabular}

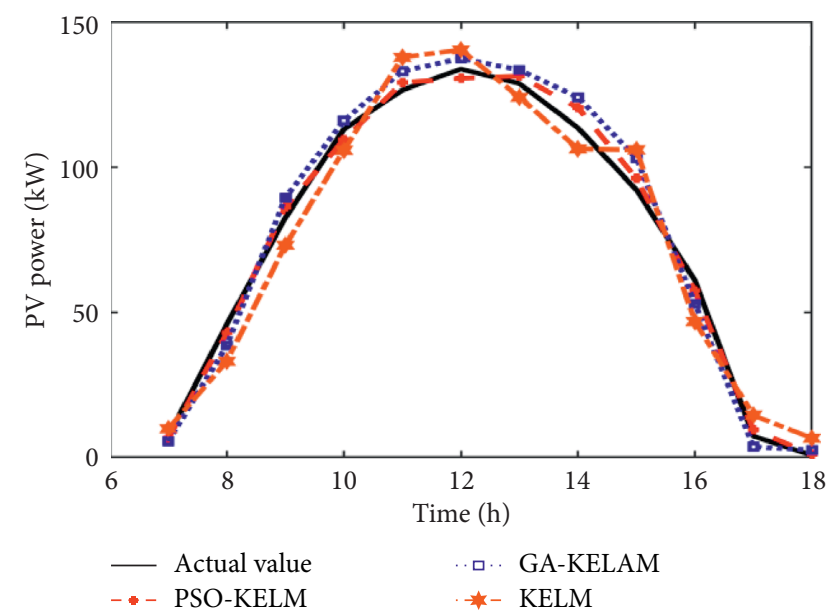

Figure 6: Prediction results of PV under different methods.

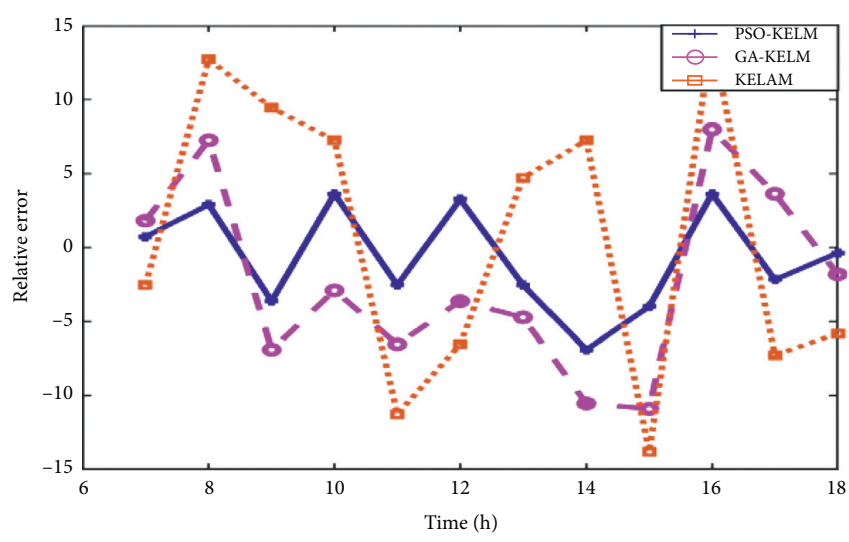

FIgURE 7: Relative errors of PV under different methods.

Table 2 shows the load forecasting results of the three forecasting models in working days. PSO-KELM prediction model has a good prediction effect on working day load. Taking into account the difference in electrical load demand of different day types, PSO-KELM, GA-KELM, and KELM were also used to predict the load of different day types such as weekends and holidays. Table 3 shows the load forecasting results for different day types.

As can be seen from the Table 1, PSO-KELM has the best prediction effect and the highest accuracy. The reliability of the proposed PSO-KELM algorithm is verified.

As can be seen from Table 3, among the three prediction models, PSO-KELM has the best prediction effect for different day types, while KELM has the worst prediction accuracy. At the same time, it can be seen that different forecasting models have a good effect on the load forecasting of weekends and rest days and a relatively poor accuracy on the load forecasting of May day holidays. The main reason is that the electrical load on the rest day is similar to that on the working day and has its periodic change rule. On the one hand, holiday load is affected by various uncertain factors, such as human activities, and its variation regularity is poor. On the other hand, it is caused by the lack of data collection for this type in training samples and the insufficient extraction of sample characteristic values by the prediction model.

5.3. Performance Test of MSFLA. In order to verify the performance of the proposed MSFLA, four test functions are selected for simulation verification. The selected test functions are shown in Table 4, and the test results are listed in Table 5.

The four test functions are, respectively, measured by SFLA, PSO, and MSFLA. The population size of the three algorithms and the total number of maximum iterations are set as 100 and 1000 . Other parameters are as follows: the size of SFLA and MSFLA subgroups is $N=30$, the number of iterations of the subgroup is 10, the MSFLA variation probability constants are $k_{1}=0.01$ and $k_{2}=0.1$, the inertia weights of PSO are $w_{1}=0.9$ and $w_{2}=0.4$, and the learning factor is $c_{1}=c_{2}=2$. In order to reduce the random error in the simulation process and test the performance of the algorithm, the search times of each group of test functions are set as 20 , and the average value of the operation results is taken. The simulation results are shown in Figure 10 and Table 5.

It can be seen from Figure 10 and Table 5 that the optimization results presented by the three algorithms are different. MSFLA is superior to SFLA and PSO in terms of average optimal value, optimal solution, and standard deviation, which indicates that improved SFLA has higher optimization precision and better algorithm stability.

5.4. Comprehensive Energy Optimization Scheduling Based on PSO-KELM and MSFLA. In this paper, it is assumed that the rated power of the micro gas turbine (MT) is $100 \mathrm{~kW}$, the power generation efficiency is 0.4 , and the heat dissipation coefficient is 0.1 . The equipment climbing and descending amount is $80 \mathrm{~kW}$, and the start-stop cost is $5 \mathrm{CNY}$. The total capacity of the pumped storage power station (PS) reservoir is $350 \mathrm{~kW}$. The maximum charge-discharge power is $50 \mathrm{~kW}$. The charge-discharge efficiency is 0.95 , and the self-loss rate is 0.0025 . Heat storage tank (HS) capacity is set to $300 \mathrm{~kW}$. Maximum heat storage and release power is $50 \mathrm{~kW}$. Heat storage efficiency is 0.8 , and heat release efficiency is 0.9 . Selfloss rate is set to 0.003 . The cooling coefficient and heating coefficient of the absorption refrigerator (AC) are 1.2 and 0.8 , respectively. The refrigeration coefficient of electric refrigerator (EC) is 4.3, and the maximum refrigeration power is $150 \mathrm{~kW}$. The maximum heat generation power of gas-fired boiler (GB) is $300 \mathrm{~kW}$, and the heat generation coefficient is 0.95 . The climbing and descending amount of equipment is $100 \mathrm{~kW}$, and the start-stop cost is 5 yuan. The number of optimized cycles is $T=24$. 


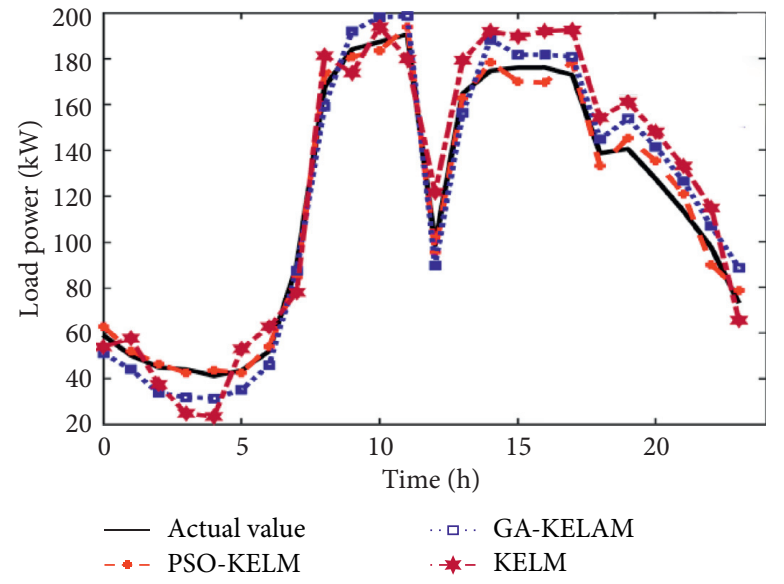

(a)

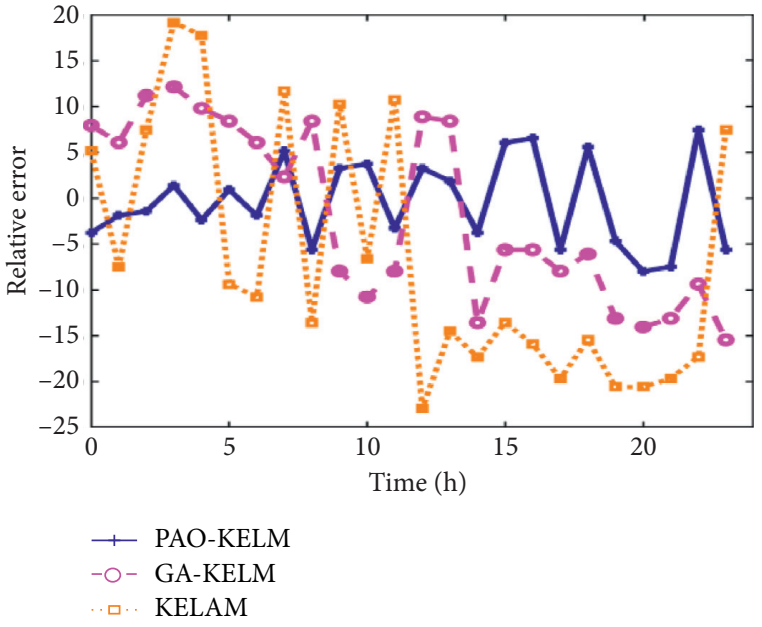

(b)

FIGURE 8: Comparison of load prediction and relative error.

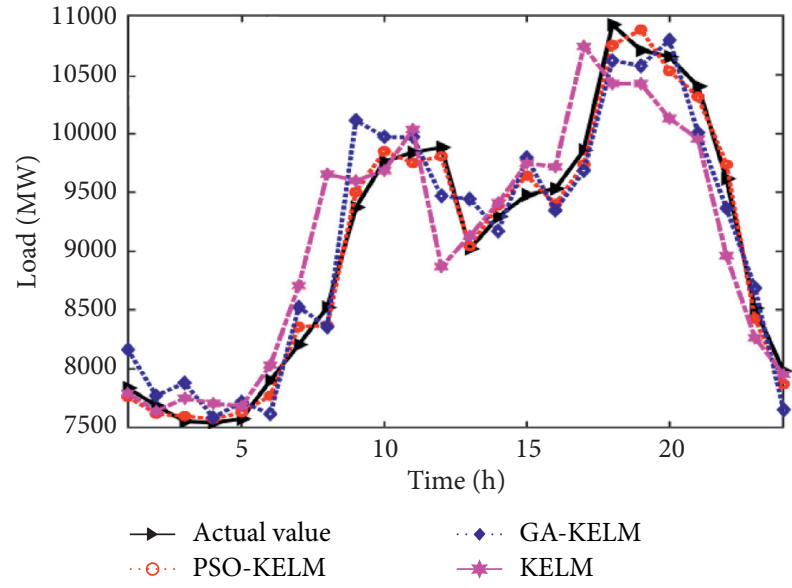

(a)

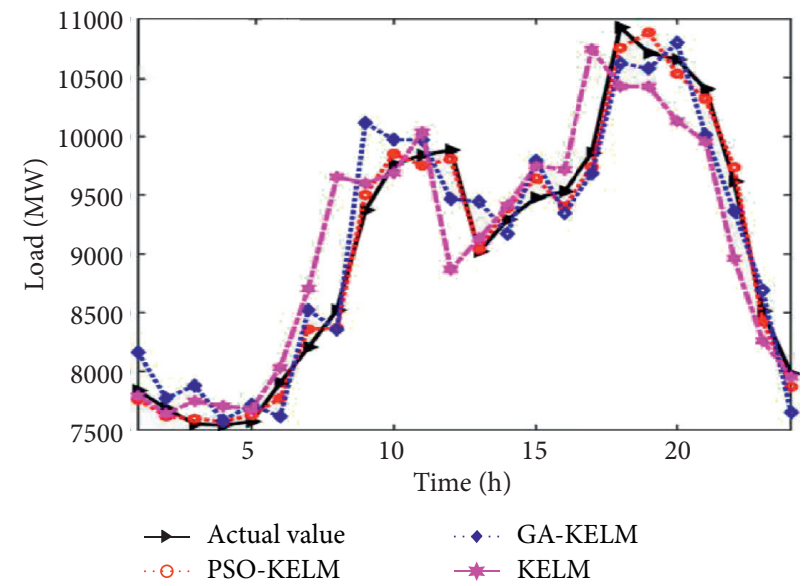

(b)

FIgURE 9: Comparison of relative error.

The cost coefficient of equipment operation and maintenance is shown in Table 6 . The time-segment energy information prices are shown in Table 7.

Due to the difference in load demand of cold, heat, and electricity in summer and winter, there are different requirements for optimal dispatching of CCHP microgrid. The typical days in summer and winter are analyzed, respectively. The proposed PSO-KELM algorithm is used to predict the cooling, heating, and wind power in the short term. Figure 11 shows the forecast data of cooling, heating, and electric load demand in two typical days in summer and winter, and Figure 12 shows the predicted power change curves of wind power generation and photovoltaic power generation in summer and winter.

5.4.1. Simulation of Typical Daily Optimal Scheduling in Summer. In summer, the optimized dispatch results of cooling, heating, and electricity loads are shown in Figures 13-15, respectively.
It can be seen from the electric load balance dispatching curve in Figure 13 that in order to make full use of wind power and photovoltaic power generation, new energy is absorbed to the maximum extent. Net electric load refers to the difference between the predicted electric load and the wind output at the corresponding time.

From 23:00 to 7:59 in the valley period, power is purchased from the large grid to drive the air conditioning refrigerator for refrigeration to meet the cooling load demand. At the same time, pumped storage is carried out to store the electricity during the valley period and transfer it to the peak period. At the end of 7 o 'clock, the pumped storage power station reaches the maximum energy storage state.

During peak hours of $8: 00-13: 59$ and $18: 00-20: 59$, the side purchase price of power grid is relatively high. The pumped storage power station operates with maximum output, and the remaining power shortage is made up by the microturbine. In this period, the power generation cost of 
TABle 2: Prediction results of the three prediction models in working days.

\begin{tabular}{lllcr}
\hline Prediction algorithm & MAPE & RMSE & MAE & CC \\
\hline KELM & 0.0285 & 8.2432 & 6.9744 & 0.9943 \\
GA-KELM & 0.0189 & 5.3517 & 4.4157 & 0.9974 \\
PSO-KELM & 0.0115 & 3.6756 & 2.6398 & 0.9981 \\
\hline
\end{tabular}

TABLE 3: Prediction performance evaluation of different day types by the three models.

\begin{tabular}{|c|c|c|c|c|c|c|c|c|}
\hline \multirow{2}{*}{ Prediction algorithm } & \multicolumn{4}{|c|}{ Weekends } & \multicolumn{4}{|c|}{ May day holiday } \\
\hline & MAPE & RMSE & MAE & $\mathrm{CC}$ & MAPE & RMSE & MAE & $\mathrm{CC}$ \\
\hline KELM & 0.021 & 4.6258 & 3.5368 & 0.9852 & 0.0819 & 12.9458 & 12.1041 & 0.9479 \\
\hline GA-KELM & 0.0197 & 4.6237 & 3.2549 & 0.9855 & 0.0632 & 10.5152 & 9.2097 & 0.9588 \\
\hline PSO-KELM & 0.0192 & 4.5407 & 3.1737 & 0.9857 & 0.0506 & 9.1065 & 7.2219 & 0.9631 \\
\hline
\end{tabular}

TABle 4: Standard test functions.

\begin{tabular}{lcccc}
\hline Names & Equation & Domain & Dimension & Theoretical value \\
\hline Sphere & $f=\sum_{i=1}^{n} x_{i}^{2}$ & {$[-100,100]$} & 30 & 0 \\
Rastrigin & $f=10 n+\sum_{i=1}^{n-1}\left[x_{i}^{2}-10 \cos \left(2 \pi x_{i}\right)\right]$ & {$[-5.12,5.12]$} & 30 & 0 \\
Griewank & $f=(1 / 4000) \sum_{i=1}^{n} x_{i}-\prod_{i=1}^{n} \cos \left(x_{i} / \sqrt{i}\right)+1$ & {$[-600,600]$} & 30 & 0 \\
Schafferf7 & $\left.f=\sum_{i=1}^{n-1}\left(\left(x_{i}^{2}+x_{i+1}^{2}\right)^{2}\left(\sin ^{2}\left(50\left(x_{i}^{2}+x_{i+1}^{2}\right)\right)^{0.1}\right)+1\right)\right)$ & {$[-10,10]$} & 30 & 0 \\
\hline
\end{tabular}

TABLE 5: Comparison of simulation results of three algorithms.

\begin{tabular}{lccr}
\hline Name & Algorithm & Average value & Standard deviation \\
\hline \multirow{3}{*}{ Sphere } & MSFLA & $93.9759 e-17$ & $3.483 e-17$ \\
& SFLA & $7.7265 e-11$ & $1.561 e-11$ \\
\hline \multirow{3}{*}{ Rastrigin } & PSO & $5.1878 e-06$ & $1.189 e-06$ \\
& MSFLA & $1.189 e-09$ & $1.443 e-15$ \\
& SFLA & $8.55 e-06$ & $2.227 e-06$ \\
Griewank & PSO & $5.886 e-04$ & $2.177 e-05$ \\
& MSFLA & $8.437 e-15$ & $1.272 e-15$ \\
& SFLA & $8.75 e-10$ & $2.387 e-10$ \\
Schafferf7 & PSO & 0.0197 & 0.0029 \\
& MSFLA & $1.254 e-09$ & $1.406 e-09$ \\
& SFLA & $7.722 e-06$ & $2.9579 e-06$ \\
& PSO & 0.259 & 0.000542 \\
\hline
\end{tabular}

the micro gas turbine is less than the electricity selling cost of the grid. Therefore, when the pumped storage energy and the micro gas turbine jointly generate electricity to meet the system's electrical load demand, the micro gas turbine runs at full capacity and sells the remaining electricity to the external network to earn profits. The pumped storage station discharges at the end of 13:00 and 20:00, and the reservoir energy storage reaches the lower limit.

During the peacetime period from 14:00 to 18:59, the gas-fired generator works at full capacity to meet the electrical load demand of the system, and the insufficient part purchases electricity from the grid. At the same time, in this stage, the maximum charging power is used for pumping and storing electricity to prepare energy storage for discharging in the next peak period.

By means of pumped storage, the surplus or low-price electric energy in the valley period is converted and stored and released in the peak period. In this way, the load demand is shifted, the power supply cost in peak period is reduced, and the overall economy of the microgrid system is improved.

Figures 14 and 15 are used to analyze the cold and heat energy balance of the cold-heat and power supply microgrid. In summer, the demand for cooling load is strong, while the demand for heating load is relatively small. In the period of low electricity price, the cooling load of the system is satisfied by electric refrigeration and air conditioning, and the hot load is satisfied by micro gas turbine generating power, without gas boiler output. In peacetime period from 14:00 to $18: 59$, the cooling capacity of electric refrigeration air conditioning increased. The flue gas waste heat generated by the microturbine in ordinary times is stored by the heat storage device and released and utilized in the evening peak period to reduce the comprehensive economic cost of the system. At 23:00 in the valley, due to the decrease of cold and hot loads and the influence of off-peak electricity price, 


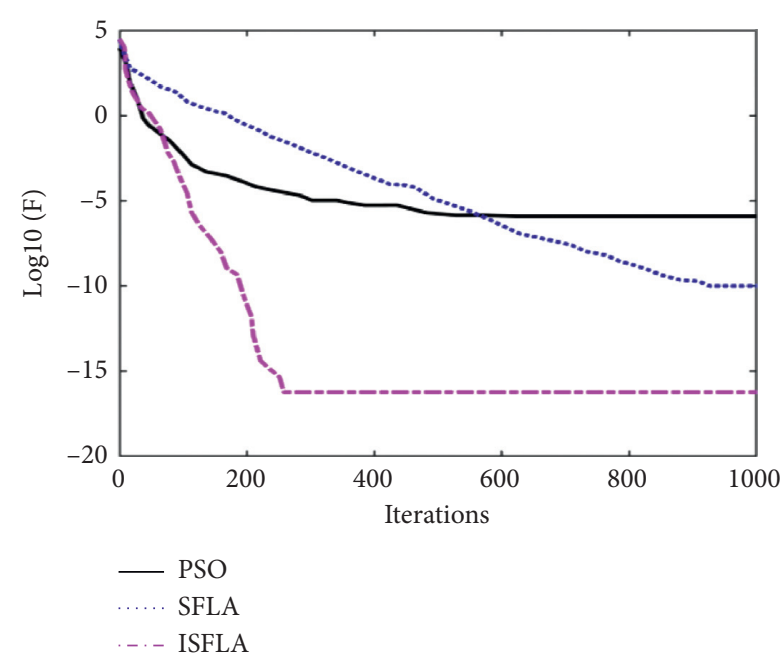

(a)

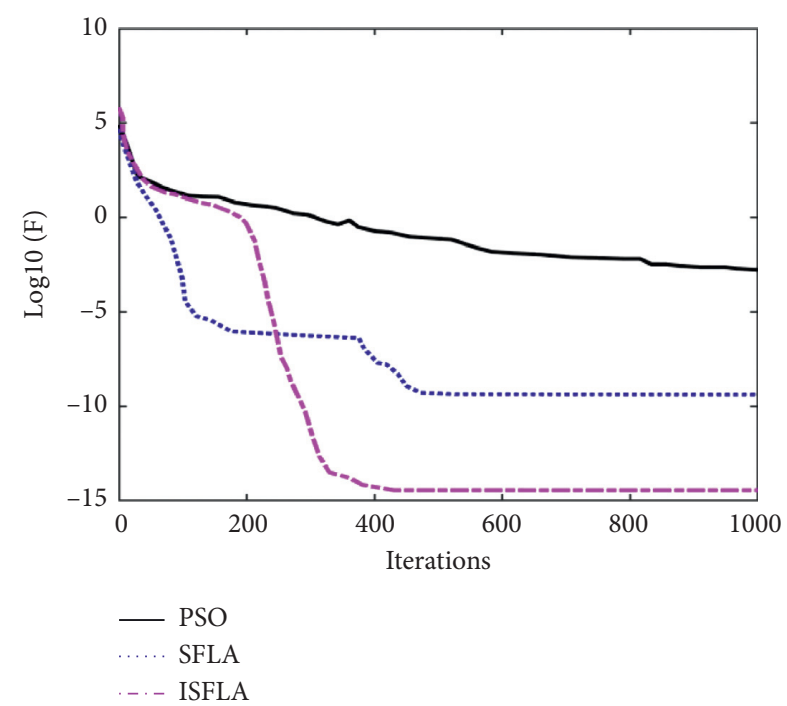

(c)

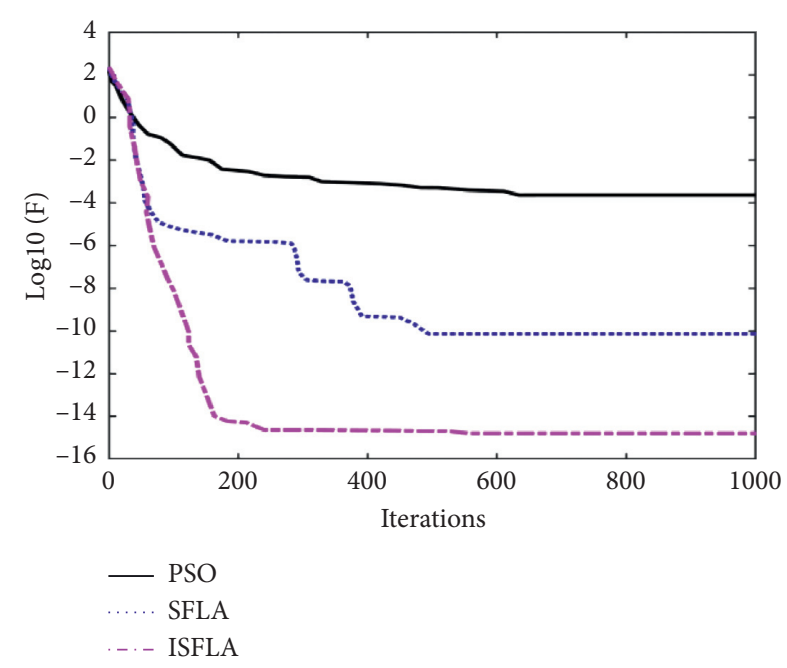

(b)

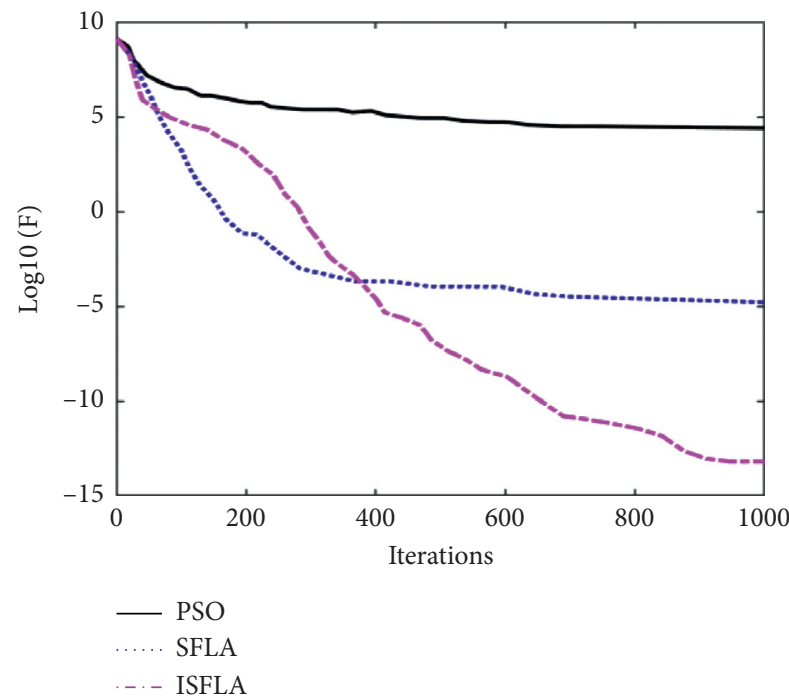

(d)

Figure 10: Optimization curves of the test functions. (a) Sphere. (b) Rastrigin. (c) Griewank. (d) Schafferf7.

TABLE 6: Maintenance coefficient of equipment operation.

\begin{tabular}{lcccccc}
\hline Equipment & PS & GB & MT & HS & AC & EC \\
\hline Maintenance cost $(\mathrm{CNY} / \mathrm{kWh})$ & 0.005 & 0.04 & 0.06 & 0.001 & 0.008 & 0.0097 \\
\hline
\end{tabular}

TABLE 7: Information table on energy prices.

\begin{tabular}{lccc}
\hline Time & Price of power purchase $(\mathrm{CNY} / \mathrm{kWh})$ & Price of selling electricity $(\mathrm{CNY} / \mathrm{k}$ Wh $)$ & Price of natural gas $\left(\mathrm{CNY} / \mathrm{m}^{3}\right)$ \\
\hline Valley period & 0.443 & 0.31 & 2.05 \\
Flat period & 0.66 & 0.506 & 2.05 \\
Peak period & 1.314 & 0.92 & 2.05 \\
\hline
\end{tabular}

the microturbine is in the state of shutdown. The cooling load is satisfied by the refrigeration air conditioning, and the hot water load is balanced by the heat release of the heat storage device.
Figure 16 shows the change curve of the energy storage state of reservoirs in pumped storage power stations. The operating costs of the cooling, heating, and power co-supply microgrid system are calculated and compared with the cost 


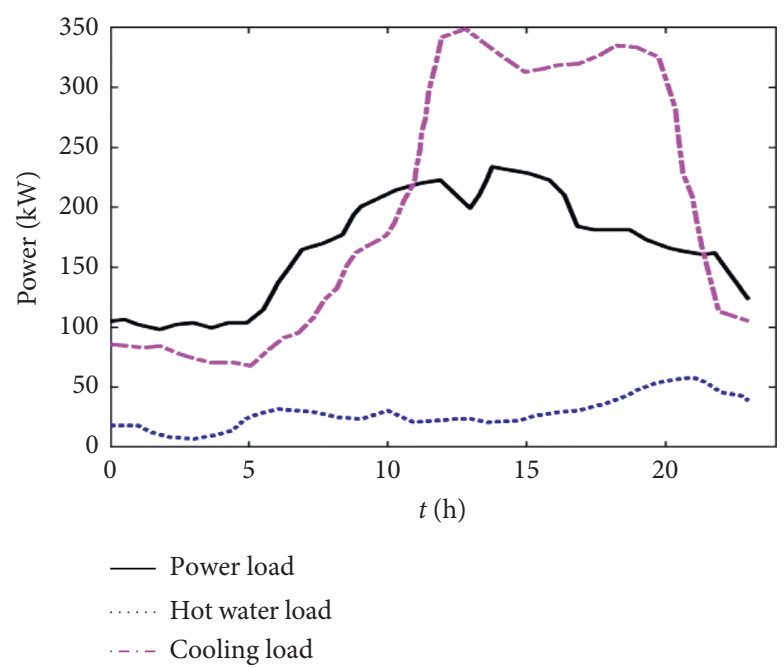

(a)

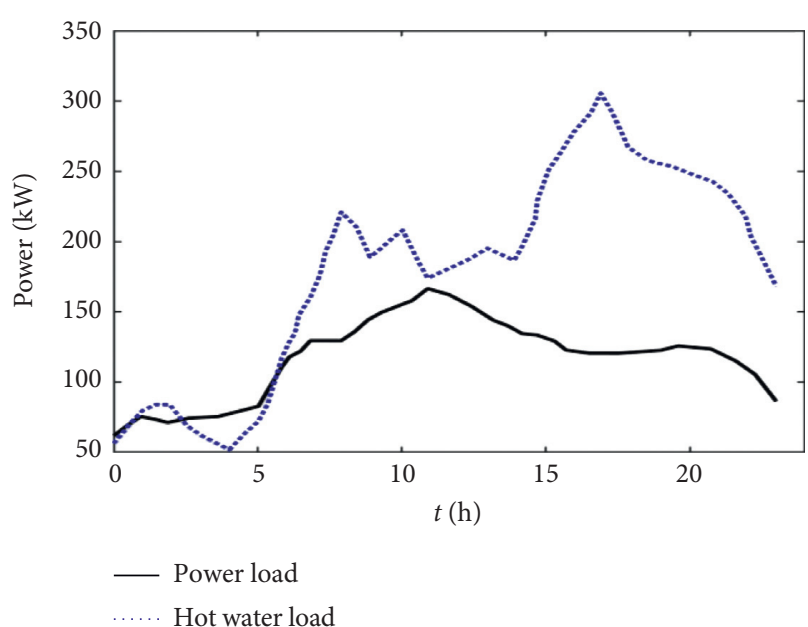

(b)

FIGURE 11: Cooling, heating, and electricity load demand curve in a typical day in summer and winter. (a) Typical load demand in summer. (b) Typical daily load demand in winter.

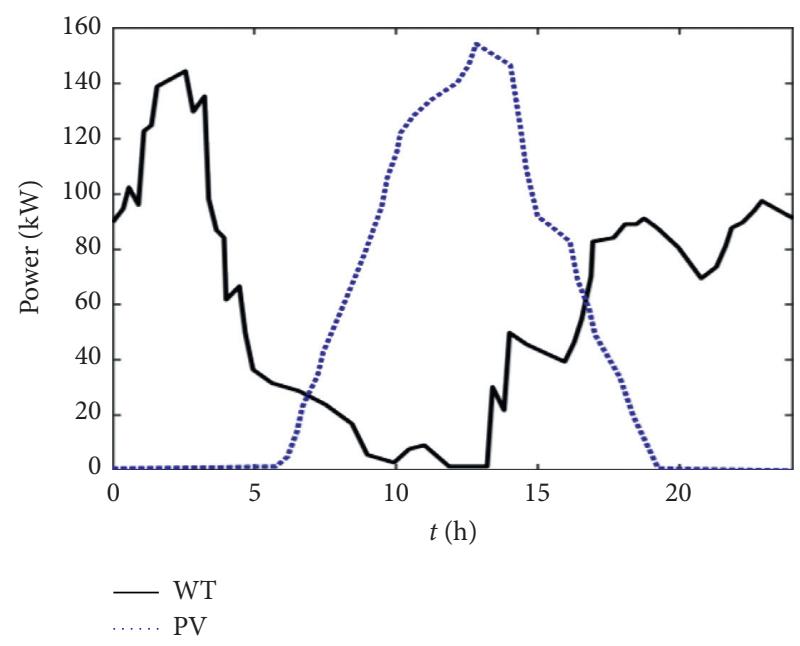

(a)

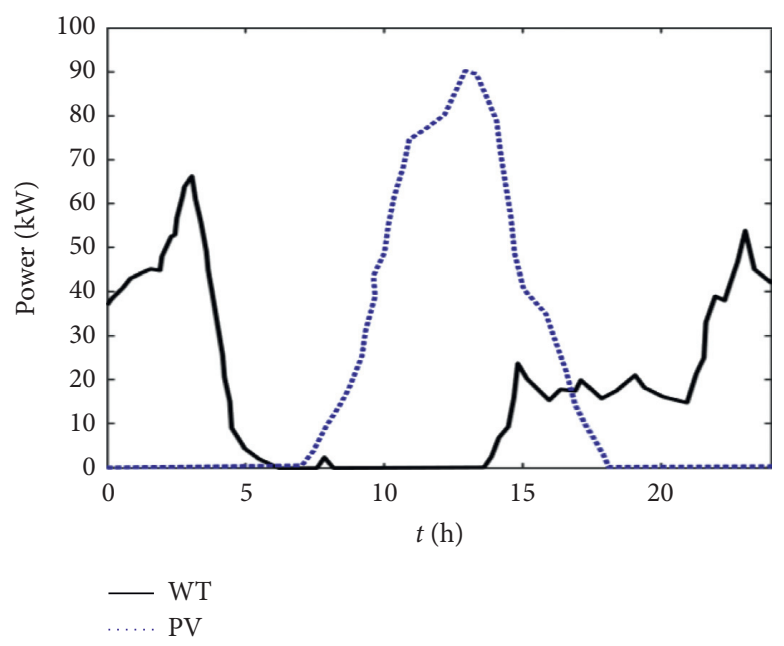

(b)

FiguRe 12: The predicted power curve of wind-solar power generation in a typical day in summer and winter. (a) Typical solar power generation in summer. (b) Typical solar power in winter.

of the traditional co-supply microgrid system. The results are shown in Table 8 and Figure 17.

It can be seen from Figure 16 that the pumped storage power station has two discharge storage processes in the whole dispatching cycle: power storage in the time periods of $1: 00-7: 59$ and $15: 00-17: 59$, discharging in the time periods of $8: 00-13: 59$ and $18: 00-21: 59$, and no o peration in the rest periods.

As can be seen from the comparison of cost data in Table 9, compared with the traditional supply division system, the power purchase cost of CCHP microgrid is reduced by $91.7 \%$, and the comprehensive operation cost is reduced by $40 \%$.

As can be seen from Figure 17, the total operating cost of CCHP system was slightly higher than that of the traditional supply distribution method in the flat-valley period. The reason is that in addition to meeting the requirements of cooling, heating, and electric load during the valley period, additional electricity needs to be purchased for energy storage. In the peak period, the operating cost of the supply mode is much higher than that of the supply mode. This is because the cost of CCHP generation is less than the selling price of the power grid, so after the pumped storage power generation and the output of the micro gas turbine meet the electrical balance of the system, the cost is reduced by selling the remaining power, and the cost is further reduced by recovering the waste heat discharged by the micro gas turbine under the full operating condition for refrigeration. In the case of split supply, electricity is purchased from the grid at a higher price to meet the electricity load and cooling 


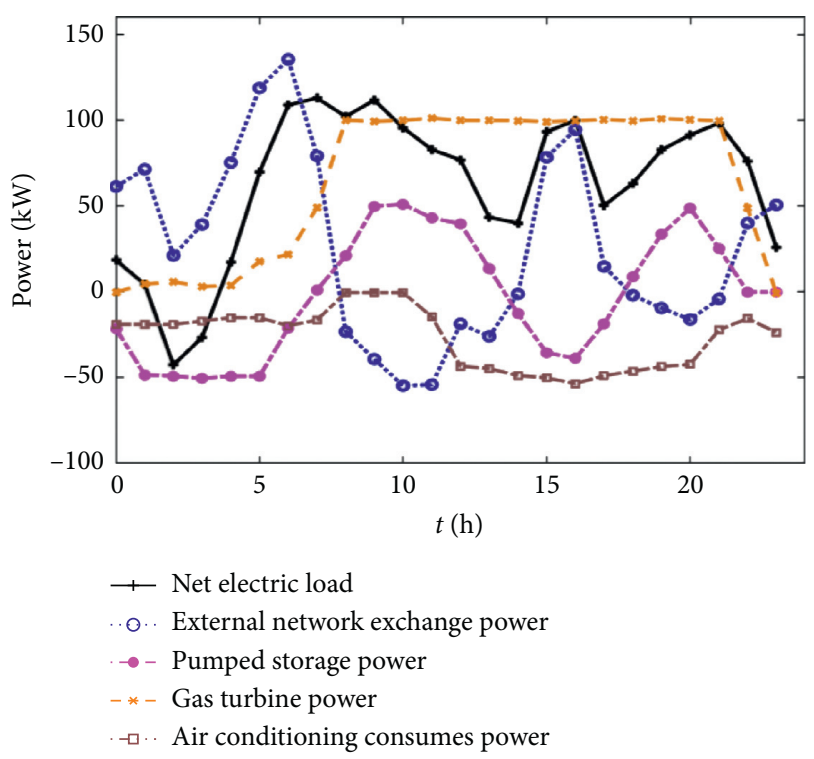

Figure 13: Typical summer equilibrium curve.

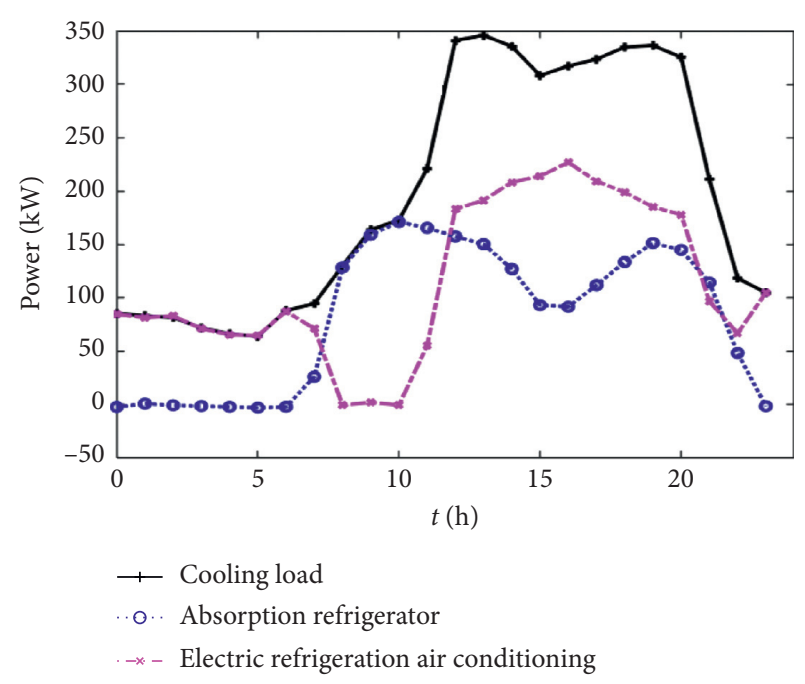

FIgURE 14: Typical diurnal cold equilibrium curve in summer.

load, which indicates that CCHP has a high economic benefit in the peak period.

5.4.2. Typical Daily Optimal Scheduling in Winter. The dispatching results of electrical load balance and thermal load balance of the CCHP microgrid on a typical day in winter are shown in Figures 18 and 19.

It can be seen from Figure 18 that compared with summer, the demand for heat energy in winter is strong, and the cooling load demand is negligible. As the core of cogeneration system, micro gas turbine is the main component of thermal load response. During the period from 00:00 to 7: 59 , the heat load demand is small, and the waste heat recovery device absorbs the waste heat from the flue gas generated during the generation process of the micro gas turbine. When the heat demand is met, the output of the micro gas turbine is increased considering the characteristics

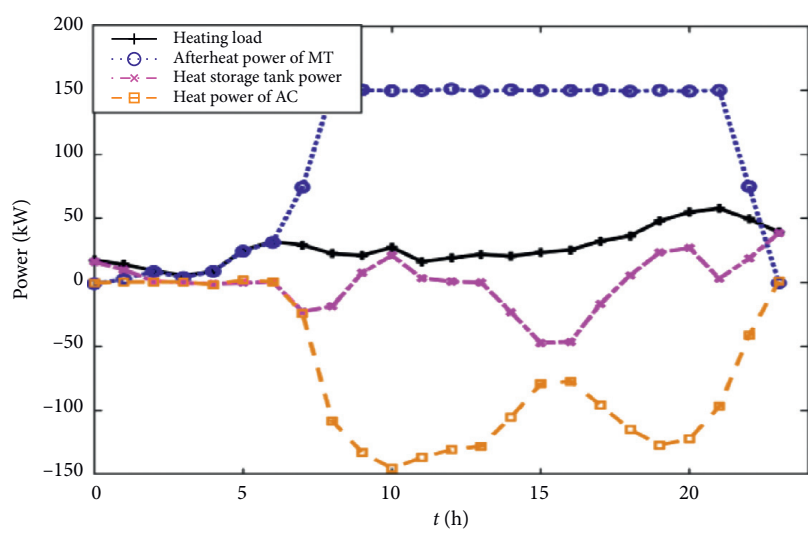

Figure 15: Typical daily heat balance curve in summer.

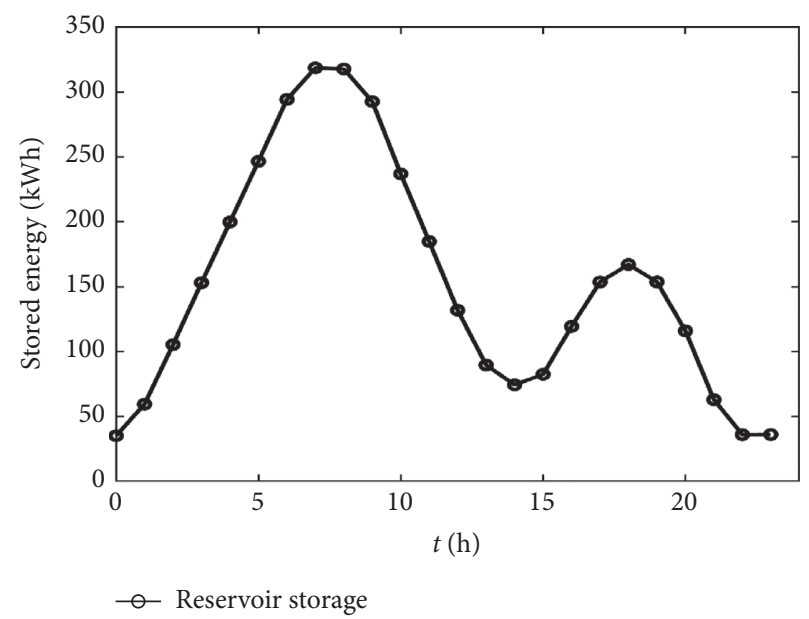

Figure 16: Energy storage curve of hydropower station.

of translational load of the storage tank, and the excess heat is stored in the storage tank. During the period from 8:00 to 23:59, with the increase of thermal load demand, the output of the micro gas turbine is increased and reaches full capacity. In this case, the heat output from the waste heat recovery unit cannot meet the heat load balance, and the heat release from the storage tank is first filled. When the waste heat recovery unit and the heat storage tank are not balanced, the heat load of the system is supplemented by the heating of the gas-fired boiler.

It can be seen from Figure 19 that the generation of microturbine is mainly determined by the thermal load demand balance. In the valley period, although the power generation cost of the micro gas turbine is higher than the power purchase cost, the operation cost of using the micro gas turbine to generate power to meet both the heat and power needs is lower than that of the supply method due to the high thermal load demand. Between 0:00 and 5:00, when the microturbine meets the thermal load demand and there is a surplus, the surplus electricity is used for pumped storage. During peak hours, the micro gas turbine reaches full capacity. Stimulated by the selling price of the power grid, the combined output of pumped storage and micro gas turbine not only meets the electricity demand but also sells 
TABLE 8: Comparison of operating cost between cold, heat, and power supply and power supply.

\begin{tabular}{lccccc}
\hline Energy method & $f_{\text {om }}(\mathrm{CNY})$ & $f_{\text {fuel }}(\mathrm{CNY})$ & $f_{\text {grid }}(\mathrm{CNY})$ & $f_{\text {open }}(\mathrm{CNY})$ & Total $(\mathrm{CNY})$ \\
\hline Combined supply of cooling & 121.47 & 1359.67 & 208.91 & 10 & 1700.05 \\
Separate power & 72.16 & 229.18 & 2522.68 & 10 & 2834.02 \\
Cost saving & -49.31 & -1130.49 & 2313.77 & 0 & 1133.97 \\
\hline
\end{tabular}

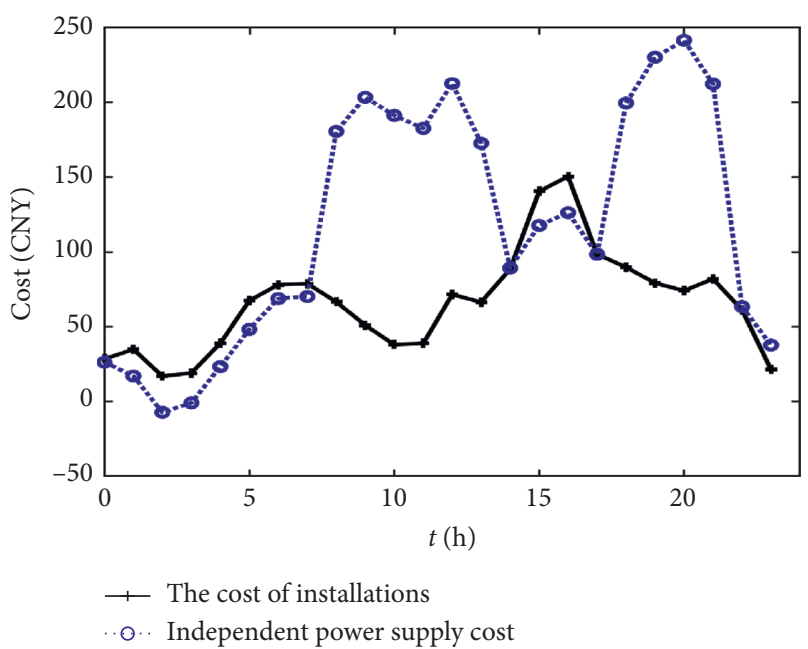

FIGURE 17: Comparison between the joint supply system and the subsupply system.

TABLE 9: Comparison of operating costs of cold, heat, and power supply combined with supply in winter.

\begin{tabular}{lccccc}
\hline Energy method & $\begin{array}{c}f_{\text {om }} \\
(\mathrm{CNY})\end{array}$ & $\begin{array}{c}f_{\text {fuel }} \\
(\mathrm{CNY})\end{array}$ & $\begin{array}{c}f_{\text {grid }} \\
(\mathrm{CNY})\end{array}$ & $\begin{array}{c}f_{\text {open }} \\
(\mathrm{CNY})\end{array}$ & $\begin{array}{c}\text { Total } \\
(\mathrm{CNY})\end{array}$ \\
\hline $\begin{array}{l}\text { Combined supply of } \\
\text { cooling }\end{array}$ & 186.59 & 2336.15 & -247.94 & 15 & 2289.8 \\
$\begin{array}{l}\text { Separate power } \\
\text { Cost saving }\end{array}$ & 174.36 & 1525.69 & 1821.96 & 10 & 3532.01 \\
\hline
\end{tabular}

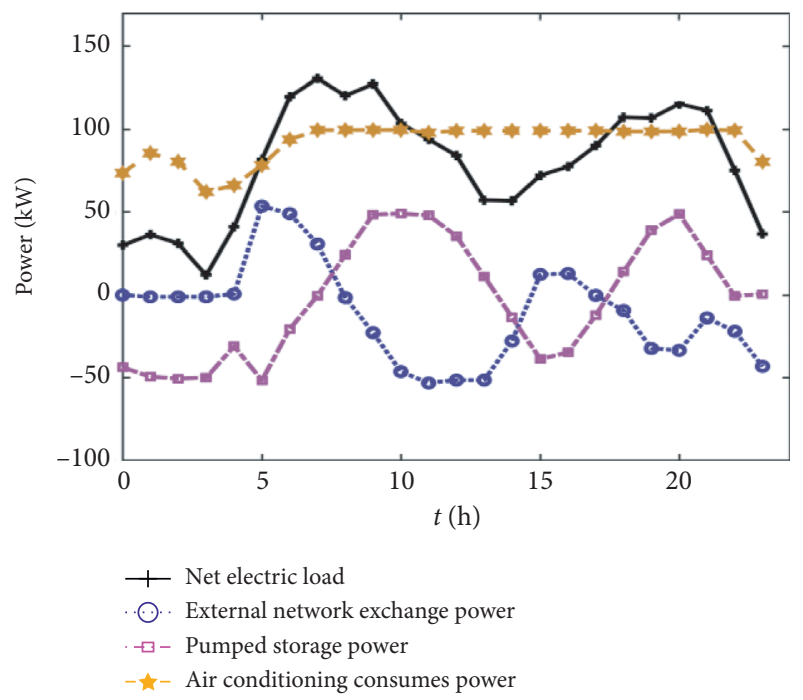

FIGURE 18: Equilibrium curve of winter conditions. more power generation to the large power grid, thus reducing the operating cost.

Figure 20 shows the change process of the reservoir energy storage state on the pumped storage power station. The comparison results of operating costs between the CCHP microgrid and the traditional supply distribution grid are shown in Table 9 and Figure 21.

It can be seen in Table 9 that compared with the traditional supply division system, although the cost of operation and maintenance and fuel consumption have been increased, the integrated operation cost of the CCHP microgrid in winter has been reduced by $35.2 \%$.

Figure 21 shows the comparison chart of the operating costs of the two modes in different periods. During the flatvalley period, the microturbine can not only meet the demand of heat and electricity load but also store the remaining heat and electricity, so the total operation cost is slightly higher than the traditional distribution method. In the peak period, the operation cost of the system is greatly reduced by using the self-generated electricity and the discharge of energy storage. In the distribution mode, electricity is purchased from the grid at a higher price to meet the electrical load demand, and a single gas-fired boiler consumes gas to provide the thermal response of the system. Therefore, the operating cost of the supply mode is much higher than that of the joint supply mode. This shows that the combined heat and power microgrid system can make full use of energy and make the overall economic benefit value higher.

5.5. Performance Comparison. In order to verify the effectiveness of the proposed method, further comparisons are made. The data used in the simulation came from an electric power company in Hubei, China. Simulations are carried out by the proposed method in this paper and the method in [30], respectively. Figure 22 shows the electric load balance dispatching curves. Figure 23 shows the thermal load balance scheduling curves. Figure 24 shows the reservoir energy storage state curve. Figure 25 shows the cost comparison curves of the proposed method and the proposed method [30]. And the cost of the two methods is listed in Table 10.

It can be seen from Figures 18, 19, 22, and 23 that, compared with the method proposed in [30], the net electrical load of the proposed method in this paper is lower than that of the external grid exchange power, and the gas turbine power and pumped storage power are higher, indicating that the proposed method has a more efficient energy utilization ratio than the method proposed in [30]. Compared with the method proposed in [30], the heat load of the proposed method in this paper is lower than that of the gas-fired 


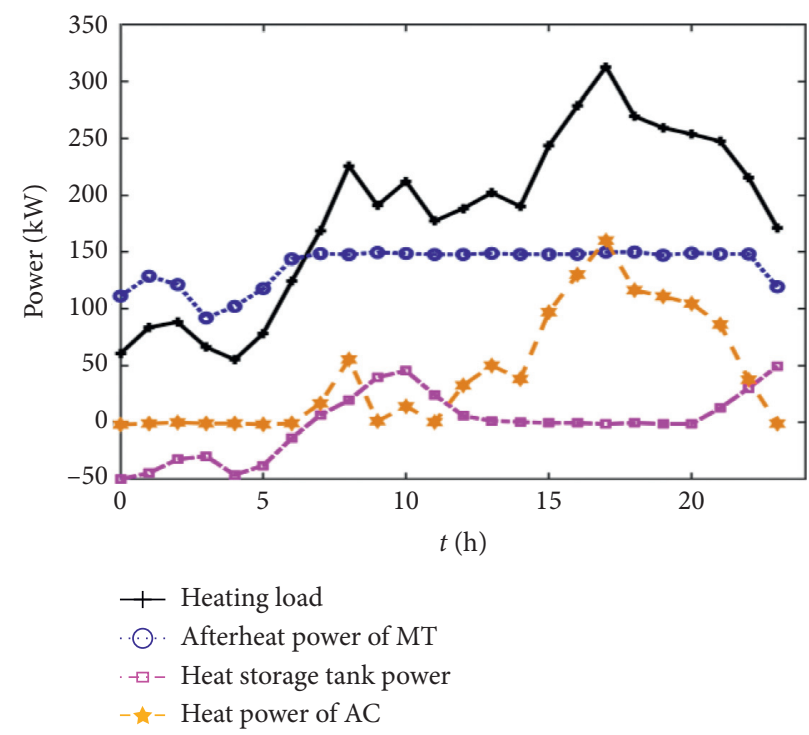

FIGURE 19: Daily heat balance curve in winter.

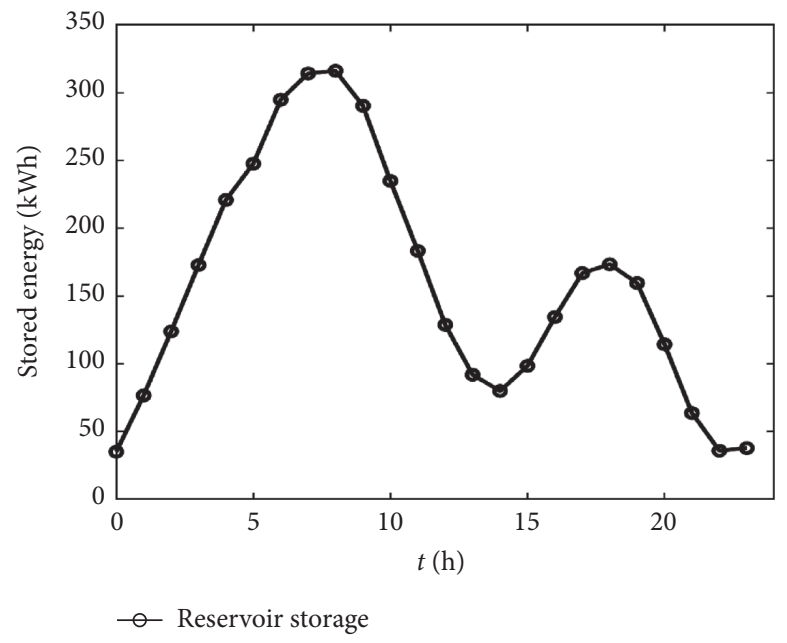

Figure 20: Energy storage of pumped storage.

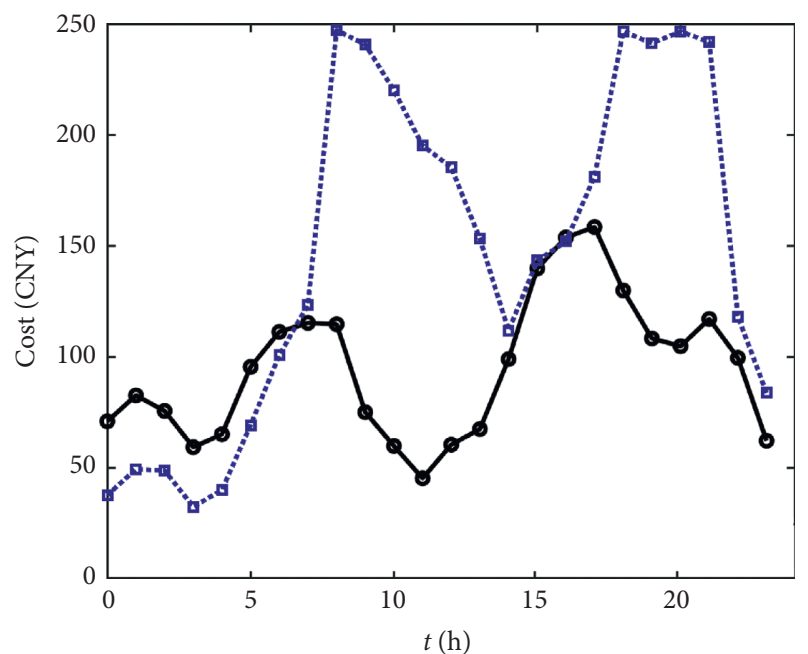

- The cost of installations

.

Figure 21: Comparison between the joint supply system and the subsupply system in winter. 


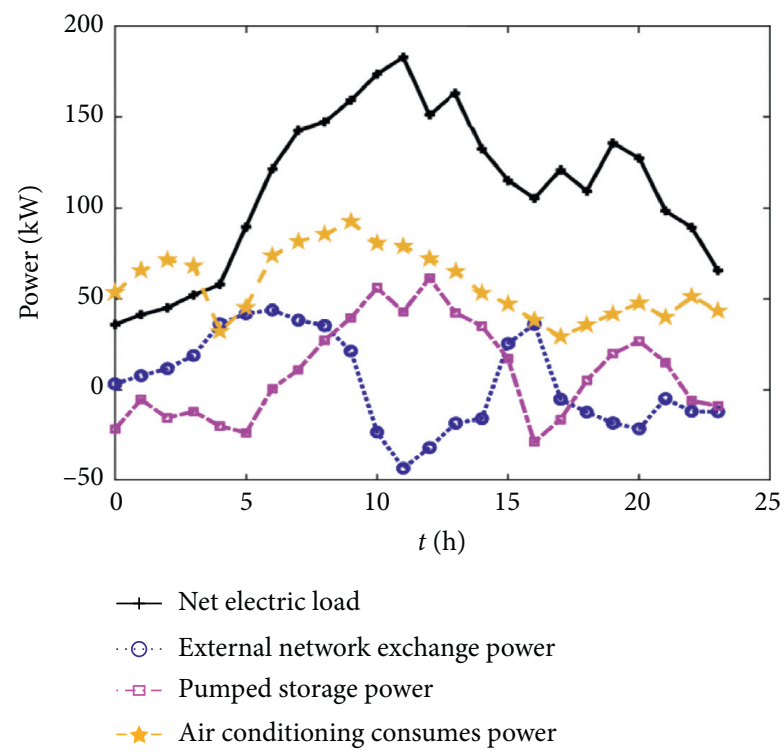

Figure 22: Equilibrium curve in winter.

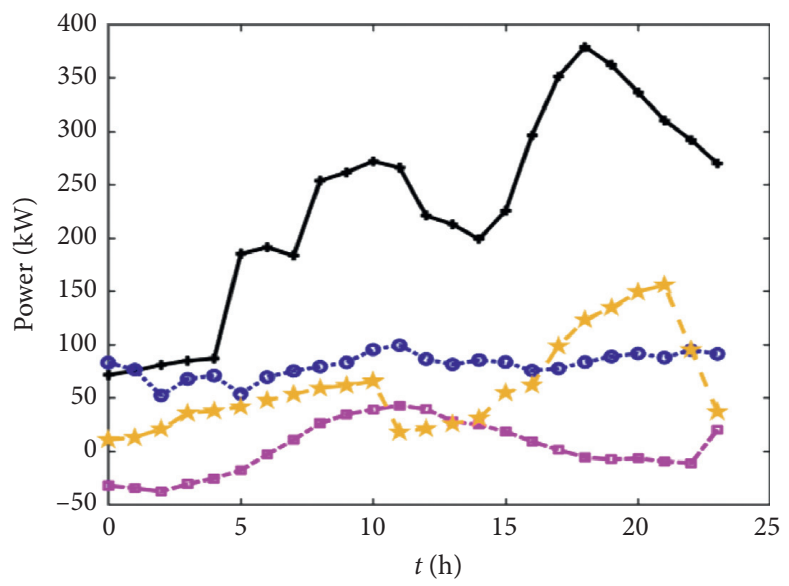

$\leftarrow$ Heating load

. ○. Afterheat power of MT

$\square-$ Heat storage tank power

- - Heat power of AC

FIgURE 23: Daily heat balance curve in winter.

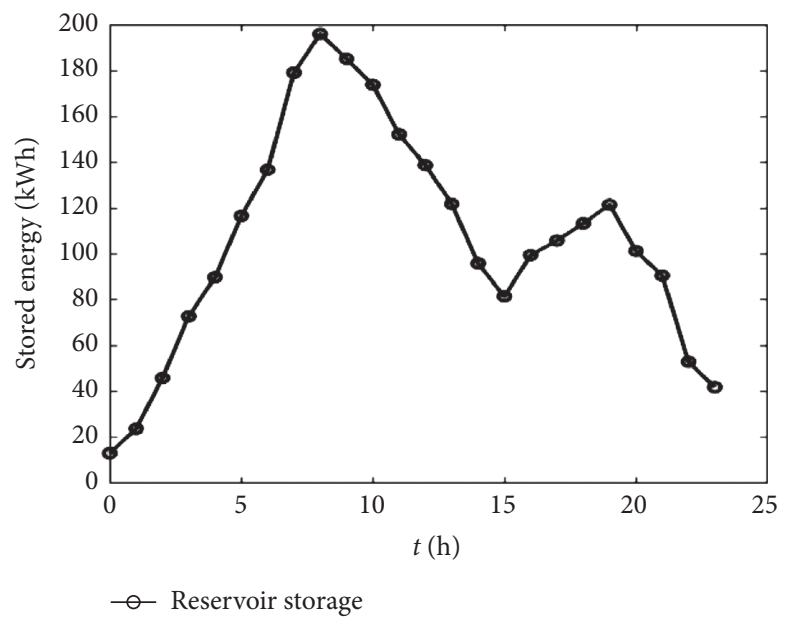

FIGURE 24: Energy storage of pumped storage in [30]. 


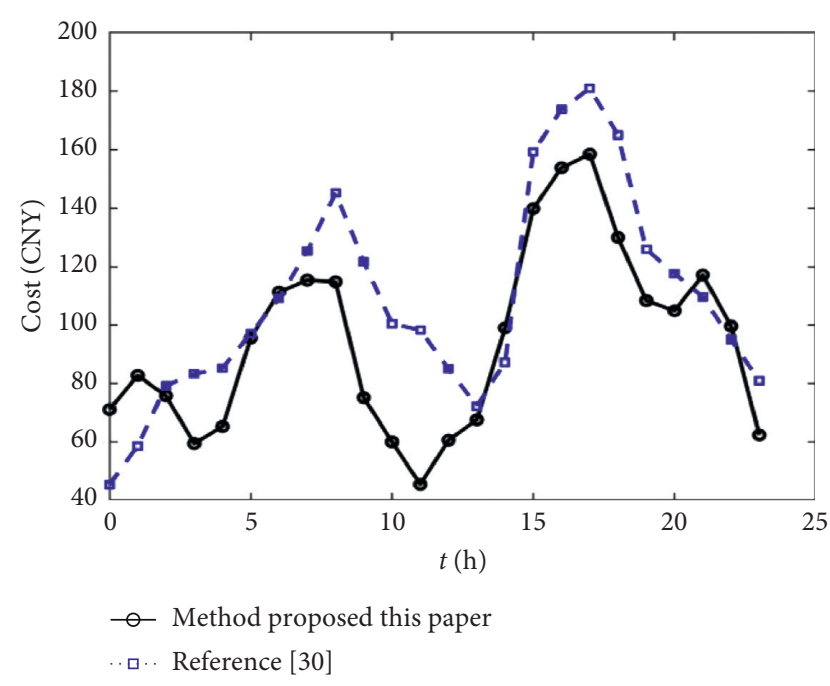

Figure 25: Comparison between the method proposed in this paper and the method in [30].

TABle 10: Daily comprehensive cost comparison results.

\begin{tabular}{|c|c|c|c|c|c|}
\hline $\begin{array}{l}\text { Energy } \\
\text { method }\end{array}$ & $\begin{array}{c}f_{\text {om }} \\
(\mathrm{CNY})\end{array}$ & $\begin{array}{c}f_{\text {fuel }} \\
(\mathrm{CNY})\end{array}$ & $\begin{array}{c}f_{\text {grid }} \\
(\mathrm{CNY})\end{array}$ & $\begin{array}{c}f_{\text {open }} \\
(\mathrm{CNY})\end{array}$ & $\begin{array}{c}\text { Total } \\
(\mathrm{CNY}) \\
\end{array}$ \\
\hline $\begin{array}{l}\text { Method } \\
\text { proposed in } \\
\text { this paper }\end{array}$ & 186.59 & 2336.15 & -247.94 & 15 & 2289.8 \\
\hline Method in [30] & 189.37 & 1828.69 & 405.39 & 13 & 2436.45 \\
\hline Cost saving & 2.78 & -507.46 & 653.33 & -2 & 146.65 \\
\hline
\end{tabular}

boiler, and the MT waste heat recovery power and the heat storage tank power are higher, which verifies that the proposed method has a more efficient energy utilization ratio than the method proposed in [30]. Comparing Figures 20 and 24, it can be found that the method proposed in this paper can store more water energy and provide more electricity for the power grid.

\section{Conclusions}

Mathematical models of PV, wind power, and consumer side loads are designed, and the factors influencing them are analyzed. Prediction models for PV, wind power, and consumer loads are established by PSO optimized KELM. PV and load power are predicted by PSO-KELM, GA-KELM, and KELM, respectively, which proves that PSO-KELM is effective and has better prediction accuracy. In order to reduce the operating cost of CCHP system and improve the energy efficiency, an improved shuffled frog leaping algorithm is used to optimize the scheduling model of CCHP system. Compared with the power supply division, the operation cost of the power grid is reduced by the proposed optimization dispatching strategy of CCHP microgrid.

Although the operating cost of the CCHP microgrid can be reduced by the method proposed in this paper, there are still problems that need to be further resolved. For example, the micro power output forecasting and load forecasting models established still need to be further improved. Improvements can be made in terms of establishing more accurate mathematical models or improving the accuracy of prediction algorithms. In the CCHP microgrid optimal dispatch function, the model can also be made more accurate.

\section{Data Availability}

The data used to support the findings of this study are included within the article.

\section{Conflicts of Interest}

The authors declare that they have no conflicts of interest.

\section{Acknowledgments}

This study was supported by the National Natural Science Foundation of China (61772520 and 61802392).

\section{References}

[1] X. Yang, K. Yao, W. Meng, and L. Yang, "Optimal scheduling of CCHP with distributed energy resources based on water cycle algorithm," IEEE Access, vol. 7, pp. 105583-105592, 2019.

[2] Q. Wang, J. Liu, Y. Hu, and X. Zhang, "Optimal operation strategy of multi-energy complementary distributed CCHP system and its application on commercial building," IEEE Access, vol. 7, pp. 127839-127849, 2019.

[3] L. . h. Wang, Q. Q. Li, M. S. Sun et al., "Robust optimisation scheduling of CCHP systems with multi-energy based on minimax regret criterion," IET Generation, Transmission \& Distribution, vol. 10, no. 9, pp. 2194-2201, 2016.

[4] F. Shen, P. Ju, M. Shahidehpour, Z. Li, C. Wang, and X. Shi, "Singular perturbation for the dynamic modeling of integrated energy systems," IEEE Transactions on Power Systems, vol. 35, no. 3, pp. 1718-1728, 2020.

[5] Z. Li, Y. Xu, S. Fang, Y. Wang, and X. Zheng, "Multiobjective coordinated energy dispatch and voyage scheduling for a multienergy ship microgrid," IEEE Transactions on Industry Applications, vol. 56, no. 2, pp. 989-999, 2020.

[6] Y. N. Wang, J. K. Wu, and X. M. Mao, "Intelligent scheduling optimization of seasonal CCHP system using rolling horizon hybrid optimization algorithm and matrix model framework," IEEE Access, vol. 6, pp. 75132-75142, 2018.

[7] S. M. Nosratabadi, R.-A. Hooshmand, E. Gholipour, and S. Rahimi, "Modeling and simulation of long term stochastic assessment in industrial microgrids proficiency considering renewable resources and load growth," Simulation Modelling Practice and Theory, vol. 75, pp. 77-95, 2017.

[8] D. R. Perez, P. B. Molina, T. G. Navarro et al., "Hybrid assessment for a hybrid microgrid: a novel methodology to critically analyse generation technologies for hybrid microgrids," Renewable Energy, vol. 157, pp. 874-887, 2020.

[9] A. Lagrangea, M. S. Martínb, A. G. Martínez et al., "Sustainable microgrids with energy storage as a means to increase power resilience in critical facilities: an application to a hospital," ElectricalPowerandEnergySystems, vol. 119, no. 5, pp. 1-12, 2020.

[10] S. M. Nosratabadi, R.-A. Hooshmand, and E. Gholipour, “A comprehensive review on microgrid and virtual power plant concepts employed for distributed energy resources 
scheduling in power systems," Renewable and Sustainable Energy Reviews, vol. 67, pp. 341-363, 2017.

[11] L. Wen, K. Zhou, S. Yang, and X. Lu, "Optimal load dispatch of community microgrid with deep learning based solar power and load forecasting," Energy, vol. 171, pp. 1053-1065, 2019.

[12] A. Sujil, R. Kumar, and R. C. Bansal, "FCM ClusteringANFIS-based PV and wind generation forecasting agent for energy management in a smart microgrid," The Journal of Engineering, vol. 2019, no. 18, pp. 4852-4857, 2019.

[13] Y. K. Semero, J. H. Zhang, and D. H. Zheng, "EMD-PSOANFIS-based hybrid approach for short-term load forecasting in microgrids," IET Generation Transmission \& Distribution, vol. 14, no. 3, pp. 470-475, 2020.

[14] N. Amjady, F. Keynia, and H. Zareipour, "Short-term load forecast of microgrids by a new bilevel prediction strategy," IEEE Transactions on Smart Grid, vol. 1, no. 3, pp. 286-294, 2020.

[15] D. Sáez, F. Ávila, D. Olivares, C. Canizares, and L. Marin, "Fuzzy prediction interval models for forecasting renewable resources and loads in microgrids," IEEE Transactions on Smart Grid, vol. 6, no. 2, pp. 548-556, 2015.

[16] C. N. Lu, H. T. Wu, and S. Vemuri, "Neural network based short term load forecasting," IEEE Transactions on Power System, vol. 8, no. 1, pp. 336-342s, 2002.

[17] Y. Wang, Q. Chen, N. Zhang, and Y. Wang, "Conditional residual modeling for probabilistic load forecasting," IEEE Transactions on Power Systems, vol. 33, no. 6, pp. 7327-7330, 2018.

[18] Y. Wang, Q. Xia, and C. Kang, "Secondary forecasting based on deviation analysis for short-term load forecasting," IEEE Transactions on Power Systems, vol. 26, no. 2, pp. 500-507, 2011.

[19] M. Rejc and M. Pantos, "Short-term transmission-loss forecast for the slovenian transmission power system based on a fuzzy-logic decision approach," IEEE Transactions on Power Systems, vol. 26, no. 3, pp. 1151-1521, 2011.

[20] Y. Wang, Q. Chen, M. Sun, C. Kang, and Q. Xia, "An ensemble forecasting method for the aggregated load with subprofiles," IEEE Transactions on Smart Grid, vol. 9, no. 4, pp. 3906-3908, 2018.

[21] W. Kong, Z. Y. Dong, D. J. Hill, F. Luo, and Y. Xu, "Shortterm residential load forecasting based on resident behaviour learning," IEEE Transactions on Power Systems, vol. 33, no. 1, pp. 1087-1088, 2018.

[22] J. Yan, H. Zhang, Y. Liu, S. Han, L. Li, and Z. Lu, "Forecasting the high penetration of wind power on multiple scales using multi-to-multi mapping," IEEE Transactions on Power Systems, vol. 33, no. 3, pp. 3276-3284, 2018.

[23] A. Safaei, Q. M. J. Wu, T. Akilan, and Y. Yang, "System-on-achip (SoC)-based hardware acceleration for an online sequential extreme learning machine (OS-ELM)," IEEE Transactions on Computer-Aided Design of Integrated Circuits and Systems, vol. 38, no. 11, pp. 2127-2138, 2019.

[24] F. Víllora, J. F. Guerrero-Martínez, M. Wegrzyn et al., "Support tool for the combined software/hardware design of on-chip ELM training for SLFF neural networks," IEEE Transactions on Industrial Informatics, vol. 12, no. 3, pp. 1114-1123, 2016.

[25] K. Ning, M. Liu, M. Dong, C. Wu, and Z. Wu, "Two efficient twin ELM methods with prediction interval," IEEE Transactions on Neural Networks and Learning Systems, vol. 26, no. 9, pp. 2058-2071, 2015.
[26] W. Cai, J. Yang, Y. Yu, Y. Song, T. Zhou, and J. Qin, "PSOELM: a hybrid learning model for short-term traffic flow forecasting," IEEE Access, vol. 8, pp. 6505-6514, 2020.

[27] H. R. O. Rocha, L. J. Silvestre, W. C. Celeste et al., "Forecast of distributed electrical generation system capacity based on seasonal micro generators using ELM and PSO," IEEE Latin America Transactions, vol. 16, no. 4, pp. 1136-1141, 2018.

[28] F. Han, C. Yang, Y.-Q. Wu et al., "A gene selection method for microarray data based on binary PSO encoding gene-to-class sensitivity information," IEEE/ACM Transactions on Computational Biology and Bioinformatics, vol. 14, no. 1, pp. 8596, 2017.

[29] A. Asrari, S. Lotfifard, and M. S. Payam, "Pareto dominancebased multiobjective optimization method for distribution network reconfiguration," IEEE Transactions on Smart Grid, vol. 7, no. 3, pp. 1401-1410, 2016.

[30] B. L. Li, P. F. Hu, and N. Zhu, "Performance analysis and optimization of a CCHP-GSHP coupling system based on quantum genetic algorithm," Sustainable Cities and Society, vol. 46, pp. 1-12, 2019. 